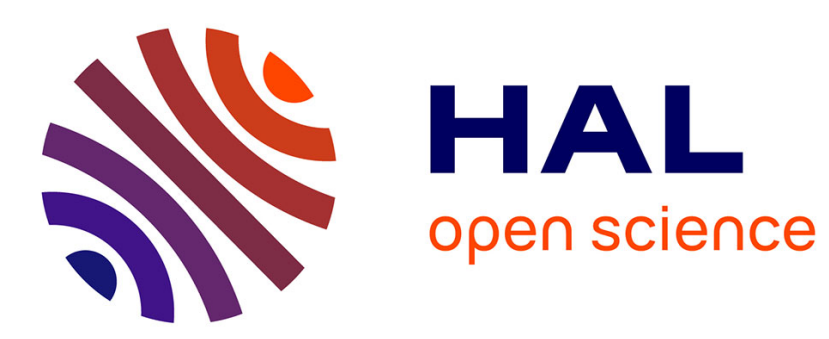

\title{
Convergence of the kinetic hydrostatic reconstruction scheme for the Saint Venant system with topography
}

François Bouchut, Xavier Lhébrard

\section{To cite this version:}

François Bouchut, Xavier Lhébrard. Convergence of the kinetic hydrostatic reconstruction scheme for the Saint Venant system with topography. Mathematics of Computation, 2021, 90 (329), pp.1119-1153. $10.1090 / \mathrm{mcom} / 3600$. hal-01515256v3

\section{HAL Id: hal-01515256 \\ https://hal.science/hal-01515256v3}

Submitted on 4 Sep 2020

HAL is a multi-disciplinary open access archive for the deposit and dissemination of scientific research documents, whether they are published or not. The documents may come from teaching and research institutions in France or abroad, or from public or private research centers.
L'archive ouverte pluridisciplinaire HAL, est destinée au dépôt et à la diffusion de documents scientifiques de niveau recherche, publiés ou non, émanant des établissements d'enseignement et de recherche français ou étrangers, des laboratoires publics ou privés. 


\title{
Convergence of the kinetic hydrostatic reconstruction scheme for the Saint Venant system with topography
}

\author{
François Bouchut* Xavier Lhébrard*
}

\begin{abstract}
We prove the convergence of the hydrostatic reconstruction scheme with kinetic numerical flux for the Saint Venant system with continuous topography with locally integrable derivative. We use a recently derived fully discrete sharp entropy inequality with dissipation, that enables us to establish an estimate in the inverse of the square root of the space increment $\Delta x$ of the $L^{2}$ norm of the gradient of approximate solutions. By Diperna's method we conclude the strong convergence towards bounded weak entropy solutions.
\end{abstract}

Keywords: Saint Venant system with topography, well-balanced scheme, hydrostatic reconstruction, convergence, entropy inequality, kinetic function.

Mathematics Subject Classification: 65M12, 76M12, 35L65

\section{Introduction and main result}

We consider the Saint Venant system

$$
\begin{aligned}
& \partial_{t} h+\partial_{x}(h u)=0, \\
& \partial_{t}(h u)+\partial_{x}\left(h u^{2}+g \frac{h^{2}}{2}\right)+g h \partial_{x} z=0,
\end{aligned}
$$

for $t \geq 0$ and $x \in \mathbb{R}$, where the unknowns are $h(t, x) \geq 0$ and $u(t, x) \in \mathbb{R}$, $g>0$ is the gravity constant, and the topography $z(x)$ is given. The system

\footnotetext{
*Université Paris-Est, Laboratoire d'Analyse et de Mathématiques Appliquées (UMR 8050), CNRS, UPEM, UPEC, F-77454, Marne-la-Vallée, France (Francois.Bouchut@u-pem.fr), (Xavier.Lhebrard@u-pem.fr)
} 
is completed with an entropy (energy) inequality

$$
\partial_{t}\left(h \frac{u^{2}}{2}+g \frac{h^{2}}{2}+g h z\right)+\partial_{x}\left(\left(h \frac{u^{2}}{2}+g h^{2}+g h z\right) u\right) \leq 0 .
$$

We shall denote $U=(h, h u)$ with $h \geq 0$, and

$$
\eta(U)=h \frac{u^{2}}{2}+g \frac{h^{2}}{2}, \quad G(U)=\left(h \frac{u^{2}}{2}+g h^{2}\right) u,
$$

the entropy and entropy fluxes without topography.

Existence and stability results for the shallow water system have been established in [27, 21, 23, 28]. Concerning approximation, many schemes have been proposed, see for example $[25,4,3,6,12,7,11,19,20]$. The hydrostatic reconstruction scheme and its variants $[4,24,18,17,16,15]$ is often used, and it is the subject of the present paper. Some results concerning consistency, stability and convergence of those schemes have been obtained in $[9,10,13,26,8,1,2]$.

In this paper we prove the convergence of the hydrostatic reconstruction scheme [4] with kinetic flux [25].

In order to explain our approach, let us consider first the case without topography. In the (time and space continuous) kinetic BGK case and without topography, the single energy inequality ensures the convergence [10]. The fully-discrete case (still without topography) is treated in [8] (a related work is [22]). Without topography the kinetic scheme can be written as a flux-vector splitting scheme

$$
U_{i}^{n+1}=U_{i}-\frac{\Delta t}{\Delta x}\left(F^{+}\left(U_{i}\right)+F^{-}\left(U_{i+1}\right)-\left(F^{+}\left(U_{i-1}\right)+F^{-}\left(U_{i}\right)\right)\right),
$$

where $F^{+}, F^{-}$are defined in (1.28). The convergence result of [8] for a scheme of the form (1.4) is established under a dissipation assumption, that $F^{+}$or $-F^{-}$is strictly $\eta$-dissipative. The $\eta$-dissipativity has been defined in [13], and analyzed in the multi-d context in [14]. Unfortunately this property that $F^{+}$ or $-F^{-}$is strictly $\eta$-dissipative does not hold for the kinetic scheme. Indeed $F^{+}$or $-F^{-}$suffer from a lack of dissipation when the state corresponds to negative (respectively positive) kinetic speeds $\xi$. Nevertheless we are able to use a weaker property, which is that $F^{+}-F^{-}$is strictly $\eta$-dissipative. When making this combination, the strict $\eta$-dissipativity corresponds to the inequality

$$
\begin{aligned}
& \int_{\mathbb{R}}|\xi|\left(H_{0}\left(M_{2}, \xi\right)-H_{0}\left(M_{1}, \xi\right)-\eta^{\prime}\left(U_{1}\right)\left(\begin{array}{l}
1 \\
\xi
\end{array}\right)\left(M_{2}-M_{1}\right)\right) d \xi \\
& \geq \alpha\left(\eta\left(U_{2}\right)-\eta\left(U_{1}\right)-\eta^{\prime}\left(U_{1}\right)\left(U_{2}-U_{1}\right)\right),
\end{aligned}
$$


for some $\alpha>0$, where $H_{0}$ is the kinetic entropy (1.12) and $M_{i}=M\left(U_{i}, \xi\right)$ with $M$ the Maxwellian equilibrium defined in (1.9). This is rigorously stated and proved in Lemma A.3 in the appendix. We have to point out however that this estimate is only valid in a closed bounded convex set which does not contain vanishing heights, and the constant $\alpha$ is not obtained explicitly.

With the inequality (1.5), the convergence proof without topography is similar to that in [8], with the additional step that one has to strengthen a bit (1.5) into an estimate in terms of $\left(M_{2}-M_{1}\right)^{2}$, as stated in Lemma A.5. This estimate is useful to take advantage of the entropy inequality

$$
\begin{aligned}
\eta\left(U_{i}^{n+1}\right) \leq & \eta\left(U_{i}\right)-\frac{\Delta t}{\Delta x}\left(\widetilde{G}_{i+1 / 2}-\widetilde{G}_{i-1 / 2}\right) \\
-\nu_{\beta} \frac{\Delta t}{\Delta x} \int_{\mathbb{R}}|\xi| \frac{g^{2} \pi^{2}}{6} & \left(\mathbb{1}_{\xi<0}\left(M_{i+1}+M_{i}\right)\left(M_{i+1}-M_{i}\right)^{2}\right. \\
& \left.+\mathbb{1}_{\xi>0}\left(M_{i}+M_{i-1}\right)\left(M_{i}-M_{i-1}\right)^{2}\right) d \xi
\end{aligned}
$$

that holds under a CFL condition, where $\widetilde{G}_{i+1 / 2}$ is a numerical entropy flux. This entropy inequality includes a dissipation term (the integral in $\xi$ ) inherited from the kinetic nature of the scheme. This term is nonnegative and measures the space variation of the unknown $U_{i}$ since $M_{i}=M\left(U_{i}, \xi\right)$. In order to get a priori estimates we sum up over the space and time indices $i$ and $n$ the previous inequality. Then we are able to use Lemma A.5, and as a consequence we get gradient estimates of the form

$$
\left\|\partial_{t} U_{\Delta}\right\|_{L_{t x}^{2}} \leq \frac{C}{\sqrt{\Delta x}}, \quad\left\|\partial_{x} U_{\Delta}\right\|_{L_{t x}^{2}} \leq \frac{C}{\sqrt{\Delta x}}
$$

where $U_{\Delta}$ is the numerical approximate solution. We conclude as in [8] by a compensated compactness argument. Indeed we recall that the compensated compactness theory $[27,23]$ gives the compactness of a bounded sequence of approximate solutions $\left(U_{\varepsilon}\right)$ which satisfy that

$$
\partial_{t} \eta_{S}\left(U_{\varepsilon}\right)+\partial_{x} G_{S}\left(U_{\varepsilon}\right) \text { is compact in } H_{l o c}^{-1},
$$

for a sufficiently large family of entropies $\eta_{S}$. According to the classical DiPerna approach [21], the estimates (1.7) are enough to establish (1.8) for all entropies.

Then, to include the topography is not an easy task, even if it is Lipschitz continuous. Indeed a typical error term produced by the scheme, corresponding to the topography term in $(1.1)$, is $\left(h_{i+1}-h_{i}\right)\left(z_{i+1}-z_{i}\right) / \Delta x$. This quantity 
is not small a priori, since $h$ is not continuous. In order to make this small one would need the compactness of $h$, that we have to prove. A key point is to use the dissipation of the discrete form of the entropy inequality (1.2). Notice that a discrete entropy inequality that puts the topography as a source term as formulated in [11] would not be sufficient because of the eventual presence of shocks.

Our convergence result with topography strongly uses the work [5], which establishes that the hydrostatic reconstruction scheme, used with the classical kinetic solver, satisfies a fully discrete entropy inequality (2.16) with dissipation but with an error term, that generalizes (1.6). However the dissipation in this inequality involves now differences $\left(M_{i+1 / 2+}-M_{i+1 / 2-}\right)^{2}$ that vanish at the lake at rest steady states, instead of previously $\left(M_{i+1}-M_{i}\right)^{2}$. Then one can simply use the triangle inequality and get $\left(M_{i+1}-M_{i}\right)^{2}$, with further error terms in $\left(z_{i+1}-z_{i}\right)^{2}$. Such error terms can be controled via Lemma A.1 and in particular (A.3). While doing such estimates one has to take care not getting cross terms $\left(h_{i+1}-h_{i}\right)\left(z_{i+1}-z_{i}\right)$ as mentioned above, that would not tend to zero. This is the main difficulty when taking into account the topography.

\subsection{Kinetic Maxwellian equilibrium}

We recall here the classical kinetic Maxwellian equilibrium, used in [25] for example. It is given by

$$
M(U, \xi)=\frac{1}{g \pi}\left(2 g h-(\xi-u)^{2}\right)_{+}^{1 / 2}
$$

where $U=(h, h u), \xi \in \mathbb{R}$ and $x_{+} \equiv \max (0, x)$ for any $x \in \mathbb{R}$. It satisfies the moment relations

$$
\int_{\mathbb{R}}\left(\begin{array}{l}
1 \\
\xi
\end{array}\right) M(U, \xi) d \xi=U, \quad \int_{\mathbb{R}} \xi^{2} M(U, \xi) d \xi=h u^{2}+g \frac{h^{2}}{2} .
$$

The interest of this particular form lies in its compatibility with a kinetic entropy given by

$$
H(f, \xi, z)=\frac{\xi^{2}}{2} f+\frac{g^{2} \pi^{2}}{6} f^{3}+g z f
$$

where $f \geq 0, \xi \in \mathbb{R}$ and $z \in \mathbb{R}$, and its version without topography

$$
H_{0}(f, \xi)=\frac{\xi^{2}}{2} f+\frac{g^{2} \pi^{2}}{6} f^{3} .
$$


Then one can check the relations

$$
\begin{array}{r}
\int_{\mathbb{R}} H(M(U, \xi), \xi, z) d \xi=\eta(U)+g h z, \\
\int_{\mathbb{R}} \xi H(M(U, \xi), \xi, z) d \xi=G(U)+g h z u,
\end{array}
$$

where $\eta$ and $G$ are given by (1.3). Moreover, for any function $f(\xi) \geq 0$, setting $h=\int f(\xi) d \xi, h u=\int \xi f(\xi) d \xi$ (assumed finite), one has the following entropy minimization principle [5],

$$
\eta(U)=\int_{\mathbb{R}} H_{0}(M(U, \xi), \xi) d \xi \leq \int_{\mathbb{R}} H_{0}(f(\xi), \xi) d \xi .
$$

Indeed this inequality is strongly related to the property (see (1.19) in [5])

$$
\partial_{f} H_{0}(M(U, \xi), \xi)=\left\{\begin{array}{l}
\eta^{\prime}(U)\left(\begin{array}{l}
1 \\
\xi
\end{array}\right) \quad \text { if } M(U, \xi)>0, \\
\geq \eta^{\prime}(U)\left(\begin{array}{l}
1 \\
\xi
\end{array}\right) \quad \text { if } M(U, \xi)=0 .
\end{array}\right.
$$

Here $\eta^{\prime}(U)$ denotes the derivative of $\eta$ with respect to $U$,

$$
\eta^{\prime}(U)=\left(g h-u^{2} / 2, u\right) .
$$

\subsection{Hydrostatic reconstruction and kinetic flux}

We consider a uniform grid $\left(x_{i+1 / 2}\right)_{i \in \mathbb{Z}}$ with space increment $\Delta x=x_{i+1 / 2}-$ $x_{i-1 / 2}$, and discrete times $t_{n}$ with a constant timestep $\Delta t, t_{n+1}-t_{n}=\Delta t$, $t_{0}=0$. We consider initial data $U^{0}=\left(h^{0}, h^{0} u^{0}\right), h^{0} \geq 0, h^{0}, u^{0} \in L^{\infty}(\mathbb{R})$ and a topography $z(x)$ assumed continuous. We define the discretization of the initial data as

$$
U_{i}^{0}=\frac{1}{\Delta x} \int_{x_{i-1 / 2}}^{x_{i+1 / 2}} U^{0}(y) d y
$$

and

$$
z_{i} \text { an approximation of } z\left(x_{i}\right),
$$

where $x_{i}=\left(x_{i+1 / 2}+x_{i-1 / 2}\right) / 2$. The hydrostatic reconstruction scheme writes [4]

$$
U_{i}^{n+1}=U_{i}^{n}-\frac{\Delta t}{\Delta x}\left(F_{i+1 / 2-}-F_{i-1 / 2+}\right),
$$

with

$$
F_{i+1 / 2-}=\mathcal{F}\left(U_{i+1 / 2-}, U_{i+1 / 2+}\right)-S_{i+1 / 2-},
$$




$$
F_{i+1 / 2+}=\mathcal{F}\left(U_{i+1 / 2-}, U_{i+1 / 2+}\right)+S_{i+1 / 2+},
$$

with $\mathcal{F}$ is a numerical flux for the system without topography. The source terms $S_{i+1 / 2-}, S_{i+1 / 2+}$ are defined by

$$
S_{i+1 / 2-}=\left(\begin{array}{c}
0 \\
\frac{g}{2} h_{i+1 / 2-}^{2}-\frac{g}{2} h_{i}^{2}
\end{array}\right), \quad S_{i+1 / 2+}=\left(\begin{array}{c}
0 \\
\frac{g}{2} h_{i+1}^{2}-\frac{g}{2} h_{i+1 / 2+}^{2}
\end{array}\right) .
$$

The reconstructed states

$$
U_{i+1 / 2-}=\left(h_{i+1 / 2-}, h_{i+1 / 2-} u_{i}\right), \quad U_{i+1 / 2+}=\left(h_{i+1 / 2+}, h_{i+1 / 2+} u_{i+1}\right)
$$

are defined by

$$
h_{i+1 / 2-}=\left(h_{i}+z_{i}-z_{i+1 / 2}\right)_{+}, \quad h_{i+1 / 2+}=\left(h_{i+1}+z_{i+1}-z_{i+1 / 2}\right)_{+}
$$

and

$$
z_{i+1 / 2}=\max \left(z_{i}, z_{i+1}\right) .
$$

The hydrostatic reconstruction scheme is defined for arbitrary numerical flux $\mathcal{F}$, but in the present paper we are only able to analyze the kinetic flux vector splitting given by

$$
\begin{gathered}
\mathcal{F}\left(U_{l}, U_{r}\right)=F^{+}\left(U_{l}\right)+F^{-}\left(U_{r}\right), \\
F^{+}(U)=\int_{\mathbb{R}} \xi \mathbb{1}_{\xi>0}\left(\begin{array}{l}
1 \\
\xi
\end{array}\right) M(U, \xi) d \xi, \\
F^{-}(U)=\int_{\mathbb{R}} \xi \mathbb{1}_{\xi<0}\left(\begin{array}{l}
1 \\
\xi
\end{array}\right) M(U, \xi) d \xi,
\end{gathered}
$$

with $M(U, \xi)$ defined by (1.9).

We consider a velocity $v_{m} \geq 0$ such that for all $i$,

$$
M\left(U_{i}, \xi\right)>0 \Rightarrow|\xi| \leq v_{m} .
$$

This means equivalently that $\left|u_{i}\right|+\sqrt{2 g h_{i}} \leq v_{m}$. We consider a CFL condition strictly less than one,

$$
v_{m} \frac{\Delta t}{\Delta x} \leq \beta<1,
$$

where $\beta$ is a constant.

An estimate that will be useful later on is that with the definitions (1.24)(1.26) one has

$$
\begin{aligned}
& 0 \leq h_{i}-h_{i+1 / 2-} \leq\left|z_{i+1}-z_{i}\right| \\
& 0 \leq h_{i}-h_{i-1 / 2+} \leq\left|z_{i}-z_{i-1}\right|
\end{aligned}
$$




\subsection{Convergence result}

Let $\left(U_{i}^{n}, z_{i}\right)$ be defined by the scheme (1.18)-(1.28). We define the approximate solution by

$$
\begin{aligned}
& U_{\Delta}(t, x) \\
& \begin{array}{l}
=\frac{1}{\Delta t}\left[\frac{U_{i+1}^{n+1}-U_{i}^{n+1}-U_{i+1}^{n}+U_{i}^{n}}{\Delta x}\left(x-x_{i}\right)+U_{i}^{n+1}-U_{i}^{n}\right]\left(t-t_{n}\right) \\
\qquad+\frac{U_{i+1}^{n}-U_{i}^{n}}{\Delta x}\left(x-x_{i}\right)+U_{i}^{n}, \\
\text { for } x_{i}<x<x_{i+1} \text { and } t_{n} \leq t<t_{n+1},
\end{array}
\end{aligned}
$$

and we set

$$
z_{\Delta}(x)=\frac{z_{i+1}-z_{i}}{\Delta x}\left(x-x_{i}\right)+z_{i}, \quad \text { for } x_{i}<x<x_{i+1} .
$$

These formulas mean that we take the continuous piecewise affine functions in space with values $U_{i}$ (or $z_{i}$ ) at $x_{i}$, and then interpolate similarly in time between $t_{n}$ and $t_{n+1}$ to get $U_{\Delta}$. In this way $U_{\Delta}$ and $z_{\Delta}$ are continuous. We shall assume that $z$ is continuous and bounded with $L_{l o c}^{1}$ derivative, and that the values $z_{i}$ are well chosen, so that as $\Delta x \rightarrow 0$

$$
z_{\Delta} \longrightarrow z \text { locally uniformly in } \mathbb{R}, \quad \frac{\mathrm{d} z_{\Delta}}{\mathrm{d} x} \longrightarrow \frac{\mathrm{d} z}{\mathrm{~d} x} \text { in } L_{l o c}^{1}(\mathbb{R}),
$$

and for any bounded interval $[a, b]$,

$$
T V 2_{[a, b]}\left(\left(z_{i}\right)\right) \rightarrow 0
$$

where $T V 2_{[a, b]}\left(\left(z_{i}\right)\right)$ is defined as

$$
T V 2_{[a, b]}\left(\left(z_{i}\right)\right) \equiv \sum_{\left[x_{i}, x_{i+1}\right] \subset[a, b]}\left(z_{i+1}-z_{i}\right)^{2}
$$

The properties (1.35) and (1.36) hold in particular for the choice $z_{i}=z\left(x_{i}\right)$, see Lemma A.1.

Moreover, for $0<h_{m}<h_{M}$ and $u_{M}>0$, we set

$$
\mathcal{U}_{h_{m}, h_{M}, u_{M}}=\left\{(h, h u) \in \mathbb{R}^{2}, \quad h_{m} \leq h \leq h_{M}, \quad|u| \leq u_{M}\right\}
$$

which is a convex set. We state now the main result of this article, which is the convergence of the hydrostatic reconstruction scheme with kinetic numerical flux. 
Theorem 1.1. Let $U^{0}=\left(h^{0}, h^{0} u^{0}\right), h^{0} \geq 0, h^{0}, u^{0} \in L^{\infty}(\mathbb{R})$, be an initial data and let $z$ be a continuous and bounded given topography satisfying $\partial_{x} z \in$ $L_{l o c}^{1}$. Define $\left(U_{i}^{n}, z_{i}\right)$ by the scheme (1.18)-(1.28), the approximate solution $U_{\Delta}$ by (1.33) and the approximate topography $z_{\Delta}$ by (1.34). We assume that the values $z_{i}$ are well chosen, i.e. satisfy (1.35), (1.36). Then we assume to have uniform bounds far from the vacuum,

$$
\forall i, n, \quad U_{i}^{n} \in \mathcal{U}_{h_{m}, h_{M}, u_{M}}
$$

for some $0<h_{m}<h_{M}, u_{M}>0$, with $\mathcal{U}_{h_{m}, h_{M}, u_{M}}$ defined by (1.38).

Then, under the CFL condition (1.30) and the inverse CFL condition

$$
1 \leq v^{*} \frac{\Delta t}{\Delta x}
$$

for some constant $v^{*}>0$, we have that up to a subsequence, $U_{\Delta} \rightarrow U$ a.e. in $(0, T) \times \mathbb{R}$ and in $C_{t}\left([0, T], L_{w *}^{\infty}(\mathbb{R})\right)$ as $\Delta t \rightarrow 0$ and $\Delta x \rightarrow 0$, where $U$ is a weak solution to (1.1) with initial data $U^{0}$, that satisfies the entropy inequality (1.2), and the family of entropy regularity conditions

$$
\partial_{t} \eta_{S}(U)+\partial_{x} G_{S}(U) \in \mathcal{M}_{l o c}
$$

for all suitable couples entropy-entropy flux $\left(\eta_{S}, G_{S}\right)$.

Some comments on this theorem are in order. At first, a main assumption is the boundedness away from vacuum (1.39). We are not able to treat the vacuum at the present time. Also, to have $L^{\infty}$ bounds is not guaranteed a priori, since only $L^{2}$ type bounds are available, obtained by integration in time and space of the discrete entropy inequality. Indeed, $L^{\infty}$ bounds can only be proved in the context of having a large family of entropy inequalities, as in [9], while here we have only one. Note that the bound (1.29) involving $v_{m}$ can be seen as a consequence of the upper bounds $h_{M}, u_{M}$ involved in (1.39). The inverse CFL condition (1.40) is a technical assumption that ensures the finite speed of propagation: since the information propagates of at most one cell per timestep, this condition ensures that the domain of dependency remains bounded as $\Delta t$ and $\Delta x$ tend to 0 . Notice that all together, the CFL and inverse CFL conditions (1.30), (1.40) can be written

$$
\frac{1}{v^{*}} \leq \frac{\Delta t}{\Delta x} \leq \frac{\beta}{v_{m}}
$$

for some $0<\beta<1$ and $v^{*}>0$. A simple way to achieve this is to take $\Delta t / \Delta x$ constant, stricly less than $1 / v_{m}$. Another main assumption is the continuity of the topography. A discontinuous topography is not allowed, indeed in 
that case it is known that several severe difficulties arise, in particular one has non-uniqueness of solutions to the Riemann problem. Numerical issues in this situation of discontinuous topography are studied in [3, 29]. We overall assume that the topography has a locally integrable derivative, but this is a minimal assumption that enables to give sense to the product $h \partial_{x} z$ in (1.1).

We have to mention that the boundedness away from vacuum assumption (1.39) allows to bound also the reconstructed states $U_{i+1 / 2 \pm}$. Indeed according to (1.24)-(1.26) one has

$$
h_{i}-\left(z_{i+1}-z_{i}\right)_{+} \leq h_{i+1 / 2-} \leq h_{i}, \quad h_{i+1}-\left(z_{i}-z_{i+1}\right)_{+} \leq h_{i+1 / 2+} \leq h_{i+1},
$$

thus $U_{i+1 / 2 \pm} \in \mathcal{U}_{\tilde{h}_{m}, h_{M}, u_{M}}$, where $\tilde{h}_{m}$ is such that

$$
0<\tilde{h}_{m} \leq h_{m}-\sup _{i}\left|z_{i+1}-z_{i}\right|
$$

which is possible since $\sup _{i}\left|z_{i+1}-z_{i}\right| \rightarrow 0$ and thus it is lower than $h_{m}$ for $\Delta x$ small enough.

The outline of the remainder of the paper is as follows. In Section 2 we establish estimates on the gradient of the approximate solution as stated in (1.7). In Section 3 we prove some interpolation estimates. In Section 4 we finally prove Theorem 1.1. We obtain (1.8) by combining the gradient estimate and the interpolation estimate, then we apply compensated compactness. An appendix is devoted to the proof of various technical lemmas.

\section{Estimate of the gradient of the approxi- mate solution}

This section is devoted to the proof of the following estimate on the approximate solution.

Proposition 2.1. With the assumptions of Theorem 1.1, we define for all $U=(h, h u)$,

$$
|U|^{2}=h^{2}+\frac{u^{2} h^{2}}{g h_{M}}
$$

Let $N \in \mathbb{N}^{*}, T=N \Delta t, i_{0}, i_{1} \in \mathbb{Z}$ such that $i_{0} \leq i_{1}$. For all $i \leq j \in \mathbb{Z}$, we define the interval

$$
I_{i, j}^{v^{*}}=\left(x_{i-1 / 2}-v^{*} T, x_{j+1 / 2}+v^{*} T\right)
$$


Then there exists some constants $C_{1}, C_{2}, C_{3}$ such that

$$
\begin{gathered}
\sum_{n=0}^{N} \sum_{i=i_{0}}^{i_{1}-1} \Delta t\left|U_{i+1}^{n}-U_{i}^{n}\right|^{2} \leq C_{1} \\
\sum_{n=0}^{N-1} \sum_{i=i_{0}}^{i_{1}} \Delta t\left|U_{i}^{n+1}-U_{i}^{n}\right|^{2} \leq C_{1} \frac{\Delta t^{2}}{\Delta x^{2}} v_{m}^{2} \\
\left(\int_{0}^{T} \int_{x_{i_{0}-1 / 2}}^{x_{i_{1}+1 / 2}}\left|\partial_{x} U_{\Delta}\right|^{2} d x d t\right)^{1 / 2} \leq \frac{C_{2}}{\sqrt{\Delta x}} \\
\left(\int_{0}^{T} \int_{x_{i_{0}-1 / 2}}^{x_{i_{1}+1 / 2}}\left|\partial_{t} U_{\Delta}\right|^{2} d x d t\right)^{1 / 2} \leq \frac{C_{3}}{\sqrt{\Delta x}}
\end{gathered}
$$

The constants $C_{1}, C_{2}, C_{3}$ depend only on $g, h_{m}, h_{M}, u_{M}, v_{m}, \beta$, the final time $T,\|z\|_{L^{\infty}}, T V 2_{I_{i_{0}, i_{1}}^{v^{*}}}\left(\left(z_{i}\right)\right),\left\|\eta\left(U^{0}\right)\right\|_{L^{1}\left(I_{i_{0}, i_{1}}^{v^{*}}\right)}$ and $\left\|h^{0}\right\|_{L^{1}\left(I_{i_{0}, i_{1}}^{v^{*}}\right)}$.

The proof of this proposition is given below in the remainder of this section. These estimates on $\partial_{t} U_{\Delta}$ and $\partial_{x} U_{\Delta}$ use recent results on discrete kinetic inequalities established in [5]. In Subsection 2.3 we use several technical lemmas which are put in the appendix for the sake of clarity of the presentation.

\subsection{Estimate of bounded propagation for the space in- tegral of the height}

We here establish some bound on $\sum_{i=i_{0}}^{i_{1}} \Delta x h_{i}^{N}$. We have

$$
h_{i}^{n+1}=h_{i}^{n}-\frac{\Delta t}{\Delta x}\left(F_{i+1 / 2}^{h}-F_{i-1 / 2}^{h}\right) \text {, }
$$

with

$$
F_{i+1 / 2}^{h}=\int_{\mathbb{R}} \xi \mathbb{1}_{\xi>0} M\left(U_{i+1 / 2-}^{n}, \xi\right) d \xi+\int_{\mathbb{R}} \xi \mathbb{1}_{\xi<0} M\left(U_{i+1 / 2+}^{n}, \xi\right) d \xi .
$$

We recall that under the CFL condition (1.30) one has $h_{i}^{n+1} \geq 0$, see [5]. We multiply by $\Delta x$ and sum over index $i$ and we obtain

$$
\sum_{i=i_{0}}^{i_{1}} \Delta x h_{i}^{n+1}=\sum_{i=i_{0}}^{i_{1}} \Delta x h_{i}^{n}-\Delta t\left(F_{i_{1}+1 / 2}^{h}-F_{i_{0}-1 / 2}^{h}\right) .
$$


Then we notice that with (1.29) and (1.42)

$$
-F_{i_{1}+1 / 2}^{h} \leq v_{m} h_{i_{1}+1 / 2+}^{n} \leq v_{m} h_{i_{1}+1}^{n}, \quad F_{i_{0}-1 / 2}^{h} \leq v_{m} h_{i_{0}-1 / 2-}^{n} \leq v_{m} h_{i_{0}-1}^{n} .
$$

With the CFL condition (1.30) we obtain

$$
\sum_{i=i_{0}}^{i_{1}} \Delta x h_{i}^{n+1} \leq \sum_{i=i_{0}-1}^{i_{1}+1} \Delta x h_{i}^{n}
$$

Denoting $T=N \Delta t$, using the previous inequality and (1.18) we get

$$
\sum_{i=i_{0}}^{i_{1}} \Delta x h_{i}^{N} \leq \sum_{i=i_{0}-N}^{i_{1}+N} \Delta x h_{i}^{0}=\int_{x_{i_{0}-N-1 / 2}}^{x_{i_{1}+N+1 / 2}} h^{0}(x) d x .
$$

Moreover we have

$$
\begin{aligned}
& x_{i_{0}-N-1 / 2}=x_{i_{0}-1 / 2}-N \Delta x=x_{i_{0}-1 / 2}-T \frac{\Delta x}{\Delta t}, \\
& x_{i_{1}+N+1 / 2}=x_{i_{1}+1 / 2}+N \Delta x=x_{i_{1}+1 / 2}+T \frac{\Delta x}{\Delta t} .
\end{aligned}
$$

Therefore using the inverse CFL condition (1.40) we get

$$
\sum_{i=i_{0}}^{i_{1}} \Delta x h_{i}^{N} \leq \int_{x_{i_{0}-1 / 2}-T v^{*}}^{x_{i_{1}+1 / 2}+T v^{*}} h^{0}(x) d x=\left\|h^{0}\right\|_{L^{1}\left(I_{i_{0}, i_{1}}^{v^{*}}\right)}
$$

with $I_{i_{0}, i_{1}}^{v^{*}}$ defined in (2.2).

\subsection{From kinetic to macroscopic discrete entropy in- equality}

We use the notations introduced in Proposition 2.1. Under the CFL condition (1.30) we can integrate with respect to $\xi$ the kinetic entropy inequality of $[5$, Theorem 3.6] as in [5, Corollary 3.7], and we obtain

$$
\begin{aligned}
& \eta\left(U_{i}^{n+1}\right)+g z_{i} h_{i}^{n+1} \leq \eta\left(U_{i}\right)+g z_{i} h_{i}-\frac{\Delta t}{\Delta x}\left(\widetilde{G}_{i+1 / 2}-\widetilde{G}_{i-1 / 2}\right) \\
&-\nu_{\beta} \frac{\Delta t}{\Delta x} \int_{\mathbb{R}}|\xi| \frac{g^{2} \pi^{2}}{6}\left(\mathbb{1}_{\xi<0}\left(M_{i+1 / 2+}+M_{i+1 / 2-}\right)\left(M_{i+1 / 2+}-M_{i+1 / 2-}\right)^{2}\right. \\
&\left.+\mathbb{1}_{\xi>0}\left(M_{i-1 / 2+}+M_{i-1 / 2-}\right)\left(M_{i-1 / 2+}-M_{i-1 / 2-}\right)^{2}\right) d \xi \\
&+C_{\beta}\left(\frac{\Delta t}{\Delta x} v_{m}\right)^{2} g\left(\left|z_{i+1}-z_{i}\right|^{2}+\left|z_{i}-z_{i-1}\right|^{2}\right)
\end{aligned}
$$


with

$$
\widetilde{G}_{i+1 / 2}=\int_{\xi<0} \xi H\left(M_{i+1 / 2+}, \xi, z_{i+1 / 2}\right) d \xi+\int_{\xi>0} \xi H\left(M_{i+1 / 2-}, \xi, z_{i+1 / 2}\right) d \xi,
$$

$\nu_{\beta}>0$ is a dissipation constant depending only on $\beta$, and $C_{\beta} \geq 0$ is a constant depending only on $\beta$. We use here the shorthand notation $M_{i} \equiv M\left(U_{i}, \xi\right)$, $M_{i+1 / 2+} \equiv M\left(U_{i+1 / 2+}, \xi\right), M_{i+1 / 2-} \equiv M\left(U_{i+1 / 2-}, \xi\right)$.

Then we follow the computations done on the height in Subsection 2.1. We multiply by $\Delta x$, take the sum over $i$ and obtain

$$
\begin{aligned}
& \sum_{i=i_{0}}^{i_{1}} \Delta x\left(\eta\left(U_{i}^{n+1}\right)+g z_{i} h_{i}^{n+1}\right) \leq \sum_{i=i_{0}}^{i_{1}} \Delta x\left(\eta\left(U_{i}\right)+g z_{i} h_{i}\right) \\
& -\Delta t \widetilde{G}_{i_{1}+1 / 2}+\Delta t \widetilde{G}_{i_{0}-1 / 2} \\
& -\nu_{\beta} \Delta t \sum_{i=i_{0}}^{i_{1}-1} \int_{\mathbb{R}}|\xi| \frac{g^{2} \pi^{2}}{6}\left(M_{i+1 / 2+}+M_{i+1 / 2-}\right)\left(M_{i+1 / 2+}-M_{i+1 / 2-}\right)^{2} d \xi \\
& -\nu_{\beta} \Delta t \int_{\mathbb{R}}|\xi| \frac{g^{2} \pi^{2}}{6} \mathbb{1}_{\xi<0}\left(M_{i_{1}+1 / 2+}+M_{i_{1}+1 / 2-}\right)\left(M_{i_{1}+1 / 2+}-M_{i_{1}+1 / 2-}\right)^{2} d \xi \\
& -\nu_{\beta} \Delta t \int_{\mathbb{R}}|\xi| \frac{g^{2} \pi^{2}}{6} \mathbb{1}_{\xi>0}\left(M_{i_{0}-1 / 2+}+M_{i_{0}-1 / 2-}\right)\left(M_{i_{0}-1 / 2+}-M_{i_{0}-1 / 2-}\right)^{2} d \xi \\
& +2 C_{\beta} \frac{\Delta t^{2}}{\Delta x} v_{m}^{2} g \sum_{i=i_{0}-1}^{i_{1}}\left|z_{i+1}-z_{i}\right|^{2} . \quad(2.18)
\end{aligned}
$$

Since $z$ is bounded, adding if necessary a sufficiently large constant to it, we can assume that $z \geq 0$. Then we notice that according to (2.17) we have

$$
-\widetilde{G}_{i_{1}+1 / 2} \leq v_{m} \eta\left(U_{i_{1}+1 / 2+}\right)+v_{m} g h_{i_{1}+1 / 2+} z_{i_{1}+1 / 2},
$$

and

$$
\widetilde{G}_{i_{0}-1 / 2} \leq v_{m} \eta\left(U_{i_{0}-1 / 2-}\right)+v_{m} g h_{i_{0}-1 / 2-} z_{i_{0}-1 / 2} .
$$

According to the definitions (1.3) of $\eta$ and (1.24), (1.25) of the reconstructed states, and to the inequalities (1.42), one has

$$
\begin{aligned}
& \eta\left(U_{i+1 / 2+}\right)+g h_{i+1 / 2+} z_{i+1 / 2} \\
\leq & \eta\left(U_{i+1}\right)+g \frac{h_{i+1 / 2+}^{2}}{2}-g \frac{h_{i+1}^{2}}{2}+g h_{i+1 / 2+} z_{i+1 / 2} \\
\leq & \eta\left(U_{i+1}\right)+g h_{i+1} z_{i+1} .
\end{aligned}
$$

Indeed, to get the last inequality, we observe that since $h_{i+1 / 2+}=\left(h_{i+1}+\right.$ $\left.z_{i+1}-z_{i+1 / 2}\right)_{+}$, either $h_{i+1}+z_{i+1}-z_{i+1 / 2} \leq 0$, which implies that $h_{i+1 / 2+}=0$ 
and the desired inequality, or $h_{i+1}+z_{i+1}-z_{i+1 / 2} \geq 0$, which implies

$$
\begin{aligned}
& g \frac{h_{i+1 / 2+}^{2}}{2}-g \frac{h_{i+1}^{2}}{2}+g h_{i+1 / 2+} z_{i+1 / 2} \\
\leq & g h_{i+1 / 2+}\left(h_{i+1 / 2+}-h_{i+1}+z_{i+1 / 2}\right) \\
\leq & g h_{i+1} z_{i+1} .
\end{aligned}
$$

Similarly to (2.21) one has

$$
\eta\left(U_{i-1 / 2-}\right)+g h_{i-1 / 2-} z_{i-1 / 2} \leq \eta\left(U_{i-1}\right)+g h_{i-1} z_{i-1} .
$$

Using (2.21), (2.23) in (2.19), (2.20) leads to

$$
\begin{gathered}
-\Delta t \widetilde{G}_{i_{1}+1 / 2} \leq \Delta t v_{m}\left(\eta\left(U_{i_{1}+1}\right)+g h_{i_{1}+1} z_{i_{1}+1}\right), \\
\Delta t \widetilde{G}_{i_{0}-1 / 2} \leq \Delta t v_{m}\left(\eta\left(U_{i_{0}-1}\right)+g h_{i_{0}-1} z_{i_{0}-1}\right) .
\end{gathered}
$$

Neglecting in (2.18) the two boundary integrals and using (2.24),(2.25), we obtain

$$
\begin{aligned}
& \sum_{i=i_{0}}^{i_{1}} \Delta x\left(\eta\left(U_{i}^{n+1}\right)+g z_{i} h_{i}^{n+1}\right) \leq \sum_{i=i_{0}-1}^{i_{1}+1} \Delta x\left(\eta\left(U_{i}\right)+g z_{i} h_{i}\right) \\
&-\nu_{\beta} \Delta t \sum_{i=i_{0}}^{i_{1}-1} \int_{\mathbb{R}}|\xi| \frac{g^{2} \pi^{2}}{6}\left(M_{i+1 / 2+}+M_{i+1 / 2-}\right)\left(M_{i+1 / 2+}-M_{i+1 / 2-}\right)^{2} d \xi \\
&+2 C_{\beta} \frac{\Delta t^{2}}{\Delta x} v_{m}^{2} g \sum_{i=i_{0}-1}^{i_{1}}\left|z_{i+1}-z_{i}\right|^{2} .
\end{aligned}
$$

Iterating (2.26) from $n=N-1$ to $n=0$ and using that

$$
\begin{gathered}
\sum_{n=0}^{N-1} 2 C_{\beta} \frac{\Delta t^{2}}{\Delta x} v_{m}^{2} g \sum_{i=i_{0}-N}^{i_{1}+N}\left|z_{i+1}-z_{i}\right|^{2} \\
\leq 2 C_{\beta} T \frac{v_{m} \Delta t}{\Delta x} v_{m} g T V 2_{\left[x_{i_{0}-N}, x_{i_{1}+N}\right]}\left(\left(z_{i}\right)\right),
\end{gathered}
$$

and that according to (1.40) one has $N \Delta x \leq v^{*} N \Delta t=v^{*} T$, we get

$$
\begin{aligned}
& \sum_{i=i_{0}}^{i_{1}} \Delta x\left(\eta\left(U_{i}^{N}\right)+g z_{i} h_{i}^{N}\right) \\
& +\nu_{\beta} \sum_{n=0}^{N-1} \Delta t \sum_{i=i_{0}}^{i_{1}-1} \int_{\mathbb{R}}|\xi| \frac{g^{2} \pi^{2}}{6}\left(M_{i+1 / 2+}^{n}+M_{i+1 / 2-}^{n}\right)\left(M_{i+1 / 2+}^{n}-M_{i+1 / 2-}^{n}\right)^{2} d \xi \\
& \quad \leq \sum_{i=i_{0}-N}^{i_{1}+N} \Delta x\left(\eta\left(U_{i}^{0}\right)+g z_{i} h_{i}^{0}\right)+C T V 2_{\left[x_{i_{0}}-v^{*} T, x_{i_{1}}+v^{*} T\right]}\left(\left(z_{i}\right)\right), \quad
\end{aligned}
$$


with $C$ depending on $g, T, \beta, v_{m}$.

We are going to show next that the integral in the LHS of (2.28) is underestimated by a term proportionnal to $\sum_{n=0}^{N-1} \sum_{i=i_{0}}^{i_{1}-1} \Delta t\left|U_{i+1 / 2+}^{n}-U_{i+1 / 2-}^{n}\right|^{2}$.

\subsection{Lower estimate of dissipation terms}

We first notice that

$$
\begin{aligned}
& \int_{\mathbb{R}}|\xi| \frac{g^{2} \pi^{2}}{6}\left(M_{i+1 / 2+}^{n}+M_{i+1 / 2-}^{n}\right)\left(M_{i+1 / 2+}^{n}-M_{i+1 / 2-}^{n}\right)^{2} d \xi \\
& \geq \frac{1}{2} \int_{\mathbb{R}}|\xi| \frac{g^{2} \pi^{2}}{6}\left(2 M_{i+1 / 2+}^{n}+M_{i+1 / 2-}^{n}\right)\left(M_{i+1 / 2+}^{n}-M_{i+1 / 2-}^{n}\right)^{2} d \xi .
\end{aligned}
$$

Now according to Lemma A.5, there exists a constant $C>0$ depending only on $g, \tilde{h}_{m}, h_{M}, u_{M}$ such that

$$
\begin{aligned}
& \int_{\mathbb{R}}|\xi| \frac{g^{2} \pi^{2}}{6}\left(2 M_{1}+M_{2}\right)\left(M_{1}-M_{2}\right)^{2} d \xi \\
\geq & C\left(g \frac{\left(h_{2}-h_{1}\right)^{2}}{2}+\tilde{h}_{m} \frac{\left(u_{2}-u_{1}\right)^{2}}{2}\right)
\end{aligned}
$$

for all $U_{1}, U_{2} \in \mathcal{U}_{\tilde{h}_{m}, h_{M}, u_{M}}$, where $M_{1}=M\left(U_{1}, \xi\right), M_{2}=M\left(U_{2}, \xi\right)$. We notice that from (1.42), (1.43) we have

$$
U_{i+1 / 2+}^{n}, U_{i+1 / 2-}^{n} \in \mathcal{U}_{\tilde{h}_{m}, h_{M}, u_{M}} .
$$

Thus from (2.29) and applying the last estimate (2.30) with $U_{1}=U_{i+1 / 2+}^{n}$ and $U_{2}=U_{i+1 / 2-}^{n}$, we get

$$
\begin{array}{r}
\int_{\mathbb{R}}|\xi| \frac{g^{2} \pi^{2}}{6}\left(M_{i+1 / 2+}^{n}+M_{i+1 / 2-}^{n}\right)\left(M_{i+1 / 2+}^{n}-M_{i+1 / 2-}^{n}\right)^{2} d \xi \\
\geq C_{5}\left|U_{i+1 / 2+}^{n}-U_{i+1 / 2-}^{n}\right|^{2}
\end{array}
$$

where $C_{5}>0$ depends only on $g, h_{m}, \tilde{h}_{m}, h_{M}, u_{M}$, and $|\cdot|$ is defined in (2.1).

\subsection{Estimate of the discrete gradient}

Now we use (2.32) in (2.28) and get

$$
\begin{aligned}
& \nu_{\beta} C_{5} \sum_{n=0}^{N-1} \sum_{i=i_{0}}^{i_{1}-1} \Delta t\left|U_{i+1 / 2+}^{n}-U_{i+1 / 2-}^{n}\right|^{2} \\
\leq & \sum_{i=i_{0}-N}^{i_{1}+N} \Delta x\left(\eta\left(U_{i}^{0}\right)+g z_{i} h_{i}^{0}\right)-\sum_{i=i_{0}}^{i_{1}} \Delta x\left(\eta\left(U_{i}^{N}\right)+g z_{i} h_{i}^{N}\right) \\
& +C T V 2_{\left[x_{i_{0}}-v^{*} T, x_{i_{1}}+v^{*} T\right]}\left(\left(z_{i}\right)\right),
\end{aligned}
$$


with $C$ depending on $g, T, \beta, v_{m}$. Next, using (1.18) we have

$$
g z_{i} h_{i}^{0} \leq \frac{1}{\Delta x} \int_{x_{i-1 / 2}}^{x_{i+1 / 2}} g\|z\|_{\infty} h^{0}(x) d x
$$

and by convexity of $\eta$

$$
\eta\left(U_{i}^{0}\right) \leq \frac{1}{\Delta x} \int_{x_{i-1 / 2}}^{x_{i+1 / 2}} \eta\left(U^{0}(x)\right) d x .
$$

Summing over $i$ we obtain

$$
\sum_{i=i_{0}-N}^{i_{1}+N} \Delta x\left(\eta\left(U_{i}^{0}\right)+g z_{i} h_{i}^{0}\right) \leq \int_{x_{i_{0}-N-1 / 2}}^{x_{i_{1}+N+1 / 2}}\left(\eta\left(U^{0}(x)\right)+g\|z\|_{\infty} h^{0}(x)\right) d x .
$$

We notice that $x_{i_{0}-N-1 / 2}=x_{i_{0}-1 / 2}-N \Delta x=x_{i_{0}-1 / 2}-T \frac{\Delta x}{\Delta t}$, and according to the finite propagation hypothesis (1.40) we deduce that

$$
\begin{aligned}
\sum_{i=i_{0}-N}^{i_{1}+N} \Delta x\left(\eta\left(U_{i}^{0}\right)+g z_{i} h_{i}^{0}\right) & \leq \int_{x_{i_{0}-1 / 2}-v^{*} T}^{x_{i_{1}+1 / 2}+v^{*} T}\left(\eta\left(U^{0}(x)\right)+g\|z\|_{\infty} h^{0}(x)\right) d x \\
& =\left\|\eta\left(U^{0}\right)\right\|_{L^{1}\left(I_{i_{0}, i_{1}}^{v^{*}}\right)}+g\|z\|_{\infty}\left\|h^{0}\right\|_{L^{1}\left(I_{i_{0}, i_{1}}^{v^{*}}\right)}, \quad \text { (2.37) }
\end{aligned}
$$

with $I_{i_{0}, i_{1}}^{v^{*}}$ defined in (2.2). In addition, according to the preliminary computation (2.15) we have

$$
-\sum_{i=i_{0}}^{i_{1}} \Delta x g z_{i} h_{i}^{N} \leq g\|z\|_{\infty} \sum_{i=i_{0}}^{i_{1}} \Delta x h_{i}^{N} \leq g\|z\|_{\infty}\left\|h^{0}\right\|_{L^{1}\left(I_{i_{0}, i_{1}}^{v^{*}}\right)} .
$$

Using $(2.37),(2.38)$ in $(2.33)$ and noticing that $\eta\left(U_{i}^{N}\right) \geq 0$, we obtain that

$$
\sum_{n=0}^{N-1} \sum_{i=i_{0}}^{i_{1}-1} \Delta t\left|U_{i+1 / 2+}^{n}-U_{i+1 / 2-}^{n}\right|^{2} \leq C
$$

where $C$ depends on $g, h_{m}, \tilde{h}_{m}, h_{M}, u_{M}, v_{m}, \beta, T,\|z\|_{L^{\infty}}, T V 2_{I_{i_{0}, i_{1}}^{v^{*}}}\left(\left(z_{i}\right)\right)$, $\left\|\eta\left(U^{0}\right)\right\|_{L^{1}\left(I_{i_{0}, i_{1}}^{v^{*}}\right)}$ and $\left\|h^{0}\right\|_{L^{1}\left(I_{i_{0}, i_{1}}^{v^{*}}\right)}$. Moreover using the triangle inequality and (2.1), (1.24)-(1.26), (1.31), (1.32), we have

$$
\begin{aligned}
& \left|U_{i+1}-U_{i}\right|^{2} \\
\leq & 3\left(\left|U_{i+1 / 2+}-U_{i+1 / 2-}\right|^{2}+\left|U_{i+1 / 2+}-U_{i+1}\right|^{2}+\left|U_{i+1 / 2-}-U_{i}\right|^{2}\right) \\
\leq & 3\left(\left|U_{i+1 / 2+}-U_{i+1 / 2-}\right|^{2}+\left(1+u_{i+1}^{2} / g h_{M}\right)\left|z_{i+1}-z_{i}\right|^{2}\right. \\
& \left.+\left(1+u_{i}^{2} / g h_{M}\right)\left|z_{i}-z_{i-1}\right|^{2}\right) .
\end{aligned}
$$


With (2.39) we get (2.3) of Proposition 2.1 (apply the inequality to the final time $T+\Delta t$ to get the sum until $n=N)$. Moreover, using (1.20), (A.100), (A.101) and (2.3), we get (2.4) of Proposition 2.1.

\subsection{End of the proof of Proposition 2.1: estimate of the gradient of the approximate solution}

Now from (1.33) we compute for $t_{n} \leq t<t_{n+1}$ and $x_{i}<x<x_{i+1}$

$$
\partial_{x} U_{\Delta}=\frac{t-t_{n}}{\Delta t} \frac{U_{i+1}^{n+1}-U_{i}^{n+1}-U_{i+1}^{n}+U_{i}^{n}}{\Delta x}+\frac{U_{i+1}^{n}-U_{i}^{n}}{\Delta x}
$$

Thus we get

$$
\int_{t_{n}}^{t_{n+1}} \int_{x_{i}}^{x_{i+1}}\left|\partial_{x} U_{\Delta}\right|^{2} d x d t \leq \frac{\Delta t}{\Delta x}\left[\left|U_{i+1}^{n+1}-U_{i}^{n+1}\right|^{2}+\left|U_{i+1}^{n}-U_{i}^{n}\right|^{2}\right] .
$$

In consequence, by using (2.3) we get (2.5) by summing over $i$ and $n$. Similarly, from (1.33) we compute for $t_{n} \leq t<t_{n+1}$ and $x_{i}<x<x_{i+1}$

$$
\partial_{t} U_{\Delta}=\frac{1}{\Delta t}\left[\frac{U_{i+1}^{n+1}-U_{i}^{n+1}-U_{i+1}^{n}+U_{i}^{n}}{\Delta x}\left(x-x_{i}\right)+U_{i}^{n+1}-U_{i}^{n}\right] .
$$

Thus

$$
\int_{t_{n}}^{t_{n+1}} \int_{x_{i}}^{x_{i+1}}\left|\partial_{t} U_{\Delta}\right|^{2} d x d t \leq \frac{\Delta x}{\Delta t}\left[\left|U_{i+1}^{n+1}-U_{i+1}^{n}\right|^{2}+\left|U_{i}^{n+1}-U_{i}^{n}\right|^{2}\right] .
$$

In consequence, by using (2.4) we get (2.6) by summing over $i$ and $n$. This concludes the proof of Proposition 2.1.

\section{Interpolation estimates}

Before going into the proof of Theorem 1.1, we give some interpolation estimates.

\subsection{Definition of interpolation functions $\widetilde{U}_{\Delta}$ and $\widetilde{F}_{\Delta}$}

We define $\widetilde{U}_{\Delta}(t, x)$ a piecewise constant function in space by

$$
\widetilde{U}_{\Delta}(t, x)=U_{i}^{n}-\frac{t-t_{n}}{\Delta x}\left(F_{i+1 / 2-}-F_{i-1 / 2+}\right)
$$


for $t_{n} \leq t<t_{n+1}, x_{i-1 / 2}<x<x_{i+1 / 2}$, with $F_{i+1 / 2-}, F_{i-1 / 2+}$ defined in (1.21), (1.22). We remark that for $x_{i-1 / 2}<x<x_{i+1 / 2}$ and $n=0, \ldots, N$,

$$
\widetilde{U}_{\Delta}\left(t_{n}, x\right)=U_{i}^{n},
$$

and thus, with (1.20), $\widetilde{U}_{\Delta}$ is continuous with respect to time. We also define $\widetilde{F}_{\Delta}(t, x)$ for $x_{i-1 / 2}<x<x_{i+1 / 2}, t_{n} \leq t<t_{n+1}$, by

$$
\begin{aligned}
\widetilde{F}_{\Delta}(t, x) & =\frac{x-x_{i-1 / 2}}{\Delta x}\left(F^{+}\left(U_{i+1 / 2-}^{n}\right)+F^{-}\left(U_{i+1 / 2+}^{n}\right)\right) \\
& +\frac{x_{i+1 / 2}-x}{\Delta x}\left(F^{+}\left(U_{i-1 / 2-}^{n}\right)+F^{-}\left(U_{i-1 / 2+}^{n}\right)\right),
\end{aligned}
$$

with $F^{+}, F^{-}$defined in (1.28), $U_{i+1 / 2-}^{n}, U_{i+1 / 2+}^{n}$ defined in (1.24). Then $\widetilde{F}_{\Delta}$ is continuous with respect to $x$ and we have

$\forall i \in \mathbb{Z} \quad \widetilde{F}_{\Delta}\left(t, x_{i+1 / 2}\right)=F^{+}\left(U_{i+1 / 2-}^{n}\right)+F^{-}\left(U_{i+1 / 2+}^{n}\right)=\mathcal{F}\left(U_{i+1 / 2-}^{n}, U_{i+1 / 2+}^{n}\right)$.

Then because of (1.21), (1.22) we have the partial differential equation

$$
\partial_{t} \widetilde{U}_{\Delta}+\partial_{x} \widetilde{F}_{\Delta}=\widetilde{S}_{\Delta}
$$

with $\widetilde{S}_{\Delta}$ piecewise constant in time and space defined by

$$
\widetilde{S}_{\Delta}(t, x)=\frac{1}{\Delta x}\left(S_{i+1 / 2-}+S_{i-1 / 2+}\right)
$$

for $t_{n} \leq t<t_{n+1}$ and $x_{i-1 / 2}<x<x_{i+1 / 2}$, with $S_{i+1 / 2-}, S_{i+1 / 2+}$ defined in (1.23).

\subsection{Estimate of $\int_{0}^{T} \int_{x_{i_{0}-1 / 2}}^{x_{i_{1}+1 / 2}}\left|U_{\Delta}-\widetilde{U}_{\Delta}\right|^{2} d t d x$}

Lemma 3.1. With the assumptions of Theorem 1.1, let $N \in \mathbb{N}^{*}, T=N \Delta t$, $i_{0}, i_{1} \in \mathbb{Z}$ such that $i_{0} \leq i_{1}$. Let $U_{\Delta}$ be the approximate solution (1.33) and $\widetilde{U}_{\Delta}$ defined by (3.1). Then

$$
\left(\int_{0}^{T} \int_{x_{i_{0}-1 / 2}}^{x_{i_{1}+1 / 2}}\left|U_{\Delta}-\widetilde{U}_{\Delta}\right|^{2} d t d x\right)^{1 / 2} \leq C \sqrt{\Delta x}
$$

with $|\cdot|$ defined by (2.1). The constant $C$ depends only on $g, h_{m}, h_{M}, u_{M}, v_{m}$,

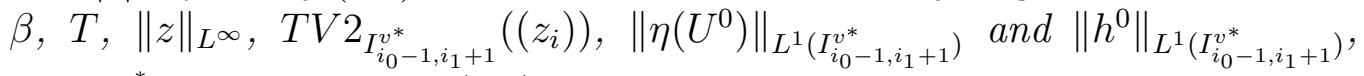
with $I_{i_{0}-1, i_{1}+1}^{v^{*}}$ defined in (2.2). 
Proof. We use the definition (1.33) of $U_{\Delta}$ and write for all $x_{i}<x<x_{i+1}$ and $t_{n} \leq t<t_{n+1}$

$$
\begin{aligned}
& U_{\Delta}-U_{i}^{n} \\
= & \frac{1}{\Delta t}\left[\frac{U_{i+1}^{n+1}-U_{i}^{n+1}-U_{i+1}^{n}+U_{i}^{n}}{\Delta x}\left(x-x_{i}\right)+U_{i}^{n+1}-U_{i}^{n}\right]\left(t-t_{n}\right) \\
& +\frac{U_{i+1}^{n}-U_{i}^{n}}{\Delta x}\left(x-x_{i}\right) .
\end{aligned}
$$

Using the triangle inequality, we obtain

$$
\left|U_{\Delta}-U_{i}^{n}\right| \leq\left|U_{i+1}^{n+1}-U_{i}^{n+1}\right|+\left|U_{i+1}^{n}-U_{i}^{n}\right|+\left|U_{i}^{n+1}-U_{i}^{n}\right| .
$$

It implies also

$$
\left|U_{\Delta}-U_{i+1}^{n}\right| \leq\left|U_{i+1}^{n+1}-U_{i}^{n+1}\right|+2\left|U_{i+1}^{n}-U_{i}^{n}\right|+\left|U_{i}^{n+1}-U_{i}^{n}\right| .
$$

Thus

$$
\begin{aligned}
& \int_{t_{n}}^{t_{n+1}} \int_{x_{i}}^{x_{i+1}}\left|U_{\Delta}-\left(U_{i}^{n} \mathbf{1}_{x_{i}<x<x_{i+1 / 2}}+U_{i+1}^{n} \mathbf{1}_{x_{i+1 / 2}<x<x_{i+1}}\right)\right|^{2} d x d t \\
\leq & 3 \Delta t \Delta x\left(\left|U_{i+1}^{n+1}-U_{i}^{n+1}\right|^{2}+4\left|U_{i+1}^{n}-U_{i}^{n}\right|^{2}+\left|U_{i}^{n+1}-U_{i}^{n}\right|^{2}\right) .
\end{aligned}
$$

Next, we set

$$
U_{\Delta}^{1}(t, x)=U_{i}^{n},
$$

for $x_{i-1 / 2}<x<x_{i+1 / 2}, t_{n} \leq t<t_{n+1}$. Taking the sum over $n$ and $i$ of (3.11) and doing translations of indices, we get

$$
\begin{aligned}
\int_{0}^{T} \int_{x_{i_{0}-1 / 2}}^{x_{i_{1}+1 / 2}}\left|U_{\Delta}-U_{\Delta}^{1}\right|^{2} d x d t & \leq 15 \Delta x \sum_{n=0}^{N} \sum_{i=i_{0}-1}^{i_{1}} \Delta t\left|U_{i+1}^{n}-U_{i}^{n}\right|^{2} \\
& +3 \Delta x \sum_{n=0}^{N-1} \sum_{i=i_{0}-1}^{i_{1}} \Delta t\left|U_{i}^{n+1}-U_{i}^{n}\right|^{2}
\end{aligned}
$$

Then we use the discrete gradient estimates (2.3), (2.4) and the CFL condition (1.30) to get

$$
\int_{0}^{T} \int_{x_{i_{0}-1 / 2}}^{x_{i_{1}+1 / 2}}\left|U_{\Delta}-U_{\Delta}^{1}\right|^{2} d x d t \leq C_{2} \Delta x
$$

with $C_{2}$ a constant depending on $g, h_{m}, h_{M}, u_{M}, v_{m}, \beta, T,\|z\|_{L^{\infty}}, T V 2_{I_{i_{0}-1, i_{1}+1}^{v^{*}}}\left(\left(z_{i}\right)\right)$, $\left\|\eta\left(U^{0}\right)\right\|_{L^{1}\left(I_{i_{0}-1, i_{1}+1}^{v^{*}}\right)}$ and $\left\|h^{0}\right\|_{L^{1}\left(I_{i_{0}-1, i_{1}+1}^{v^{*}}\right)}{ }$ 
Next we use the definition (3.1) of $\widetilde{U}_{\Delta}$ and the definitions (1.20)-(1.23) and get for all $x_{i-1 / 2}<x<x_{i+1 / 2}$ and $t_{n} \leq t<t_{n+1}$

$$
\begin{aligned}
& U_{i}^{n}-\widetilde{U}_{\Delta} \\
& =\frac{t-t_{n}}{\Delta x}\left(F^{+}\left(U_{i+1 / 2-}^{n}\right)+F^{-}\left(U_{i+1 / 2+}^{n}\right)-F^{+}\left(U_{i-1 / 2-}^{n}\right)-F^{-}\left(U_{i-1 / 2+}^{n}\right)\right. \\
& \left.\left.\quad-\frac{g}{2}\left(\left(h_{i+1 / 2-}^{n}\right)^{2}-\left(h_{i}^{n}\right)^{2}+\left(h_{i}^{n}\right)^{2}-\left(h_{i-1 / 2+}^{n}\right)^{2}\right)\right)\right)
\end{aligned}
$$

with $F^{+}, F^{-}$defined in (1.28), $U_{i+1 / 2-}^{n}, U_{i+1 / 2+}^{n}$ defined in (1.24), $h_{i+1 / 2+}^{n}$, $h_{i+1 / 2-}^{n}$ defined in (1.25). Then, using that $F^{+}$and $F^{-}$are Lipschitz continuous, see (A.100) and (A.101), with the CFL condition (1.30) we obtain that there exists $C_{3}>0$, depending on $g, h_{m}, h_{M}, u_{M}$ and $v_{m}$ such that

$$
\begin{array}{r}
\left|U_{i}^{n}-\widetilde{U}_{\Delta}\right| \leq C_{3}\left(\left|U_{i+1 / 2-}^{n}-U_{i-1 / 2-}^{n}\right|+\left|U_{i+1 / 2+}^{n}-U_{i-1 / 2+}^{n}\right|\right. \\
\left.+\left|h_{i}^{n}-h_{i+1 / 2-}^{n}\right|+\left|h_{i}^{n}-h_{i-1 / 2+}^{n}\right|\right) .
\end{array}
$$

Then using an estimate similar to (2.40) we obtain

$$
\left|U_{i}^{n}-\widetilde{U}_{\Delta}\right| \leq C_{3}\left(\left|U_{i}^{n}-U_{i-1}^{n}\right|+\left|U_{i+1}^{n}-U_{i}^{n}\right|+\left|z_{i}-z_{i-1}\right|+\left|z_{i+1}-z_{i}\right|\right) .
$$

Thus

$$
\begin{aligned}
& \int_{t_{n}}^{t_{n+1}} \int_{x_{i-1 / 2}}^{x_{i+1 / 2}}\left|U_{i}^{n}-\widetilde{U}_{\Delta}\right|^{2} d t d x \\
\leq & 4 C_{3}^{2} \Delta t \Delta x\left(\left|U_{i}^{n}-U_{i-1}^{n}\right|^{2}+\left|U_{i+1}^{n}-U_{i}^{n}\right|^{2}+\left|z_{i}-z_{i-1}\right|^{2}+\left|z_{i+1}-z_{i}\right|^{2}\right) .
\end{aligned}
$$

Taking the sum over $n$ and $i$ and doing translations of indices, we get

$$
\begin{aligned}
& \int_{0}^{T} \int_{x_{i_{0}-1 / 2}}^{x_{i_{1}+1 / 2}}\left|U_{\Delta}^{1}-\widetilde{U}_{\Delta}\right|^{2} d x d t \\
\leq & 8 C_{3}^{2} \Delta x\left(\sum_{n=0}^{N-1} \sum_{i=i_{0}-1}^{i_{1}} \Delta t\left|U_{i+1}^{n}-U_{i}^{n}\right|^{2}+\sum_{n=0}^{N-1} \sum_{i=i_{0}-1}^{i_{1}} \Delta t\left|z_{i+1}-z_{i}\right|^{2}\right),
\end{aligned}
$$


with $U_{\Delta}^{1}$ defined in (3.12). Next, using the gradient estimate (2.3) we get

$$
\int_{0}^{T} \int_{x_{i_{0}-1 / 2}}^{x_{i_{1}+1 / 2}}\left|U_{\Delta}^{1}-\widetilde{U}_{\Delta}\right|^{2} d x d t \leq C_{2} \Delta x
$$

with $C_{2}$ a constant depending on $g, h_{m}, h_{M}, u_{M}, v_{m}, \beta, T,\|z\|_{L^{\infty}}, T V 2_{I_{i_{0}-1, i_{1}+1}^{v^{*}}}\left(\left(z_{i}\right)\right)$, $\left\|\eta\left(U^{0}\right)\right\|_{L^{1}\left(I_{i_{0}-1, i_{1}+1}^{v^{*}}\right)}$ and $\left\|h^{0}\right\|_{L^{1}\left(I_{i_{0}-1, i_{1}+1}^{v^{*}}\right)}$.

Finally, noticing that $U_{\Delta}-\widetilde{U}_{\Delta}=\left(U_{\Delta}-U_{\Delta}^{1}\right)+\left(U_{\Delta}^{1}-\widetilde{U}_{\Delta}\right)$, we get

$$
\begin{array}{r}
\int_{0}^{T} \int_{x_{i_{0}-1 / 2}}^{x_{i_{1}+1 / 2}}\left|U_{\Delta}-\widetilde{U}_{\Delta}\right|^{2} d t d x \\
\leq 2\left(\int_{0}^{T} \int_{x_{i_{0}-1 / 2}}^{x_{i_{1}+1 / 2}}\left|U_{\Delta}-U_{\Delta}^{1}\right|^{2} d t d x+\int_{0}^{T} \int_{x_{i_{0}-1 / 2}}^{x_{i_{1}+1 / 2}}\left|U_{\Delta}^{1}-\widetilde{U}_{\Delta}\right|^{2} d t d x\right) .
\end{array}
$$

With (3.14) and (3.20) we get (3.7), which concludes the proof.

\subsection{Estimate of $\int_{0}^{T} \int_{x_{i_{0}-1 / 2}}^{x_{i_{1}+1 / 2}}\left|F\left(U_{\Delta}\right)-\widetilde{F}_{\Delta}\right|^{2} d t d x$}

We will see later on that in order to prove compactness of the sequence $\partial_{t} \eta\left(U_{\Delta}\right)+\partial_{x} G\left(U_{\Delta}\right)$ in $H_{l o c}^{-1}$, we need an estimate on $F\left(U_{\Delta}\right)-\widetilde{F}_{\Delta}$.

Lemma 3.2. With the assumptions of Theorem 1.1, let $N \in \mathbb{N}^{*}, T=N \Delta t$, $i_{0}, i_{1} \in \mathbb{Z}$ such that $i_{0} \leq i_{1}$. Let $U_{\Delta}$ be the approximate solution (1.33) and $\widetilde{F}_{\Delta}$ defined by (3.3). Then

$$
\left(\int_{0}^{T} \int_{x_{i_{0}-1 / 2}}^{x_{i_{1}+1 / 2}}\left|F\left(U_{\Delta}\right)-\widetilde{F}_{\Delta}\right|^{2} d t d x\right)^{1 / 2} \leq C \sqrt{\Delta x}
$$

with $|\cdot|$ defined by (2.1). The constant $C$ depends only on $g, h_{m}, h_{M}, u_{M}, v_{m}$, $\beta, T,\|z\|_{L^{\infty}}, T V 2_{I_{i_{0}}^{v^{*}-1, i_{1}+1}}\left(\left(z_{i}\right)\right),\left\|\eta\left(U^{0}\right)\right\|_{L^{1}\left(I_{i_{0}-1, i_{1}+1}^{v^{*}}\right)}$ and $\left\|h^{0}\right\|_{L^{1}\left(I_{i_{0}-1, i_{1}+1}^{v^{*}}\right)}$, $I_{i_{0}-1, i_{1}+1}^{v^{*}}$ defined in $(2.2)$.

Proof. We recall here (3.3)

$$
\begin{aligned}
\widetilde{F}_{\Delta}(t, x) & =\frac{x-x_{i-1 / 2}}{\Delta x}\left(F^{+}\left(U_{i+1 / 2-}^{n}\right)+F^{-}\left(U_{i+1 / 2+}^{n}\right)\right) \\
& +\frac{x_{i+1 / 2}-x}{\Delta x}\left(F^{+}\left(U_{i-1 / 2-}^{n}\right)+F^{-}\left(U_{i-1 / 2+}^{n}\right)\right),
\end{aligned}
$$


for all $x_{i-1 / 2}<x<x_{i+1 / 2}$ and $t_{n} \leq t<t_{n+1}$. Moreover, we have

$$
F\left(U_{\Delta}\right)=F^{+}\left(U_{\Delta}\right)+F^{-}\left(U_{\Delta}\right) .
$$

Thus, using the triangle inequality, for all $x_{i-1 / 2}<x<x_{i+1 / 2}$, we get

$$
\begin{aligned}
& \left|\widetilde{F}_{\Delta}(t, x)-F\left(U_{\Delta}(t, x)\right)\right| \\
\leq & \left|F^{+}\left(U_{i+1 / 2-}^{n}\right)-F^{+}\left(U_{\Delta}\right)\right|+\left|F^{-}\left(U_{i+1 / 2+}^{n}\right)-F^{-}\left(U_{\Delta}\right)\right| \\
& +\left|F^{+}\left(U_{i-1 / 2-}^{n}\right)-F^{+}\left(U_{\Delta}\right)\right|+\left|F^{-}\left(U_{i+1 / 2+}^{n}\right)-F^{-}\left(U_{\Delta}\right)\right| .
\end{aligned}
$$

Then, using that $F^{+}$and $F^{-}$are Lipschitz continuous, see (A.100) and (A.101), we obtain that there exists $C>0$, depending on $g, h_{m}, h_{M}, u_{M}$ and $v_{m}$ such that

$$
\begin{aligned}
& \left|\widetilde{F}_{\Delta}(t, x)-F\left(U_{\Delta}\right)\right| \\
\leq & C\left(\left|U_{i+1 / 2-}^{n}-U_{\Delta}\right|+\left|U_{i+1 / 2+}^{n}-U_{\Delta}\right|+\left|U_{i-1 / 2-}^{n}-U_{\Delta}\right|+\left|U_{i-1 / 2+}^{n}-U_{\Delta}\right|\right) .
\end{aligned}
$$

Moreover using (1.24)-(1.26), (1.31), (1.32), we get

$$
\begin{aligned}
\left|\widetilde{F}_{\Delta}(t, x)-F\left(U_{\Delta}\right)\right| \leq & C\left(2\left|U_{i}^{n}-U_{\Delta}\right|+\left|U_{i+1}^{n}-U_{\Delta}\right|+\left|U_{i-1}^{n}-U_{\Delta}\right|\right. \\
& \left.+2\left|z_{i+1}-z_{i}\right|+2\left|z_{i}-z_{i-1}\right|\right) .
\end{aligned}
$$

Thus we get

$$
\begin{aligned}
& \int_{t_{n}}^{t_{n+1}} \int_{x_{i-1 / 2}}^{x_{i+1 / 2}}\left|\widetilde{F}_{\Delta}(t, x)-F\left(U_{\Delta}\right)\right|^{2} d t d x \\
\leq & C^{2} \int_{t_{n}}^{t_{n+1}} \int_{x_{i-1 / 2}}^{x_{i+1 / 2}}\left|U_{i}^{n}-U_{\Delta}\right|^{2} d t d x \\
& +C^{2} \Delta t \Delta x\left(\left|U_{i+1}^{n}-U_{i}^{n}\right|^{2}+\left|U_{i-1}^{n}-U_{i}^{n}\right|^{2}+\left|z_{i+1}-z_{i}\right|^{2}+\left|z_{i}-z_{i-1}\right|^{2}\right) .
\end{aligned}
$$

Taking the sum over $n$ and $i$ and doing translations of indices, we get

$$
\begin{array}{r}
\int_{0}^{T} \int_{x_{i_{0}-1 / 2}}^{x_{i_{1}+1 / 2}}\left|\widetilde{F}_{\Delta}(t, x)-F\left(U_{\Delta}(t, x)\right)\right|^{2} d x d t \leq C^{2} \int_{0}^{T} \int_{x_{i_{0}-1 / 2}}^{x_{i_{1}+1 / 2}}\left|U_{\Delta}-U_{\Delta}^{1}\right|^{2} d x d t \\
+C^{2} \Delta x\left(\sum_{n=0}^{N-1} \sum_{i=i_{0}-1}^{i_{1}} \Delta t\left|U_{i+1}^{n}-U_{i}^{n}\right|^{2}+\sum_{n=0}^{N-1} \sum_{i=i_{0}-1}^{i_{1}} \Delta t\left|z_{i+1}-z_{i}\right|^{2}\right) .
\end{array}
$$

Using the previous estimate (3.14) involving $U_{\Delta}-U_{\Delta}^{1}$ and the gradient estimate (2.3), we get (3.22), which concludes the proof. 


\section{Proof of Theorem 1.1}

Using (3.5) we write

$$
\partial_{t} U_{\Delta}+\partial_{x} F\left(U_{\Delta}\right)=\partial_{t}\left(U_{\Delta}-\widetilde{U}_{\Delta}\right)+\partial_{x}\left(F\left(U_{\Delta}\right)-\widetilde{F}_{\Delta}\right)+\widetilde{S}_{\Delta},
$$

with $U_{\Delta}$ defined in (1.33), $\widetilde{U}_{\Delta}$ defined in (3.1), $\widetilde{F}_{\Delta}$ defined in (3.3), and $\widetilde{S}_{\Delta}$ defined in (3.6). Note that in (4.1) all terms are locally bounded functions. We multiply (4.1) by $\eta^{\prime}\left(U_{\Delta}\right)$ and get, for any entropy-entropy flux $(\eta, G)$, the decomposition

$$
\begin{aligned}
\partial_{t} \eta\left(U_{\Delta}\right)+\partial_{x} G\left(U_{\Delta}\right) & =\eta^{\prime}\left(U_{\Delta}\right) \cdot \partial_{t}\left(U_{\Delta}-\widetilde{U}_{\Delta}\right) \\
& +\eta^{\prime}\left(U_{\Delta}\right) \cdot \partial_{x}\left(F\left(U_{\Delta}\right)-\widetilde{F}_{\Delta}\right)+\eta^{\prime}\left(U_{\Delta}\right) \cdot \widetilde{S}_{\Delta} \\
& \equiv R_{1}+M_{1}+R_{2}+M_{2}+\eta^{\prime}\left(U_{\Delta}\right) \cdot \widetilde{S}_{\Delta}
\end{aligned}
$$

with

$$
\begin{aligned}
& R_{1}=\partial_{t}\left(\eta^{\prime}\left(U_{\Delta}\right) \cdot\left(U_{\Delta}-\widetilde{U}_{\Delta}\right)\right), \\
& M_{1}=-\eta^{\prime \prime}\left(U_{\Delta}\right) \cdot \partial_{t} U_{\Delta} \cdot\left(U_{\Delta}-\widetilde{U}_{\Delta}\right), \\
& R_{2}=\partial_{x}\left(\eta^{\prime}\left(U_{\Delta}\right) \cdot\left(F\left(U_{\Delta}\right)-\widetilde{F}_{\Delta}\right)\right), \\
& M_{2}=-\eta^{\prime \prime}\left(U_{\Delta}\right) \cdot \partial_{x} U_{\Delta} \cdot\left(F\left(U_{\Delta}\right)-\widetilde{F}_{\Delta}\right) .
\end{aligned}
$$

We have using (3.22)

$$
\begin{aligned}
& \int_{0}^{T} \int_{-R}^{R}\left|\eta^{\prime}\left(U_{\Delta}\right) \cdot\left(F\left(U_{\Delta}\right)-\widetilde{F}_{\Delta}\right)\right|^{2} d x d t \\
\leq & \left\|\eta^{\prime}\left(U_{\Delta}\right)\right\|_{L^{\infty}((0, T) \times(-R, R))}^{2} \int_{0}^{T} \int_{-R}^{R}\left|F\left(U_{\Delta}\right)-\widetilde{F}_{\Delta}\right|^{2} d x d t \\
\leq & C_{R} \Delta x,
\end{aligned}
$$

thus $R_{2}$ goes to zero in $H_{l o c}^{-1}$ as $\Delta x \rightarrow 0$. Similarly, using (3.7), $R_{1}$ goes to zero in $H_{l o c}^{-1}$ as $\Delta x \rightarrow 0$. Furthermore, using (2.5) and (3.22), we have

$$
\begin{aligned}
& \int_{0}^{T} \int_{-R}^{R}\left|M_{2}\right| d x d t \\
\leq & \left\|\eta^{\prime \prime}\left(U_{\Delta}\right)\right\|_{L^{\infty}}\left(\iint\left|\partial_{x} U_{\Delta}\right|^{2} d x d t\right)^{1 / 2}\left(\iint\left|F\left(U_{\Delta}\right)-\widetilde{F}_{\Delta}\right|^{2} d x d t\right)^{1 / 2} \\
\leq & \left\|\eta^{\prime \prime}\left(U_{\Delta}\right)\right\|_{L^{\infty}} \frac{C_{2}}{\sqrt{\Delta x}} C \sqrt{\Delta x} \\
\leq & C_{R}
\end{aligned}
$$


Thus $M_{2}$ is bounded in $\mathcal{M}_{l o c}((0, T) \times \mathbb{R})$. Similarly, using (2.6) and (3.7), $M_{1}$ is bounded in $\mathcal{M}_{l o c}((0, T) \times \mathbb{R})$.

Then, the definition (3.6) of $\widetilde{S}_{\Delta}$ and the definitions (1.23) of $S_{i+1 / 2-}$, $S_{i+1 / 2+}$ yield with (1.31), (1.32) and the $L_{l o c}^{1}$ condition (1.35) that $\widetilde{S}_{\Delta}$ is uniformly bounded in $L_{l o c}^{1}$. According to (4.2) and (4.3) one has

$$
\partial_{t} \eta\left(U_{\Delta}\right)+\partial_{x} G\left(U_{\Delta}\right)-R_{1}-R_{2}=M_{1}+M_{2}+\eta^{\prime}\left(U_{\Delta}\right) \cdot \widetilde{S}_{\Delta} .
$$

The right-hand side is bounded in $\mathcal{M}_{l o c} \cap W_{l o c}^{-1, p}, \forall p, 1<p<+\infty$, as a consequence it is compact in $H_{l o c}^{-1}$. At this point, we know that $R_{1}+R_{2}$ and $M_{1}+M_{2}+\eta^{\prime}\left(U_{\Delta}\right) \cdot \widetilde{S}_{\Delta}$ are compact in $H_{l o c}^{-1}$, therefore their sum, which is equal to $\partial_{t} \eta\left(U_{\Delta}\right)+\partial_{x} G\left(U_{\Delta}\right)$, is compact in $H_{l o c}^{-1}$. This holds for any couple entropyentropy flux $(\eta, G)$. Furthermore, $\left(U_{\Delta}\right)_{\Delta>0}$ is bounded since we assume that $\left(U_{i}^{n}\right)_{i, n}$ is a bounded sequence. We are now able to apply the compensated compactness method [23] and we get that up to a subsequence $U_{\Delta} \rightarrow U$ a.e. and in $L_{l o c, t, x}^{1}$ as $\Delta t \rightarrow 0$ and $\Delta x \rightarrow 0$.

Moreover, according to Lemma A.8, $\partial_{t} U_{\Delta}$ is bounded in $L_{t}^{\infty}\left(\mathcal{D}_{x}^{\prime}\right)$ and therefore we deduce that $U_{\Delta} \rightarrow U$ in $C_{t}\left([0, T], L_{x, w *}^{\infty}\left(\mathbb{R}_{l o c}\right)\right)$, by the Arzelà Ascoli theorem. Then, knowing that $U_{\Delta}$ converges in $L_{l o c}^{p}$ to $U$, we can apply Lemma A.9, which concludes the convergence of the approximate source term $\widetilde{S}_{\Delta}$ to $S$.

Finally we pass to the limit in (4.1) using (3.7), (3.22), which enables us to get that the limit $U$ is a weak solution to our system (1.1). Moreover passing to the limit weakly in (2.16) using (1.36), we get (1.2). Similarly, the weak limit of (4.6) yields (1.41). This ends the proof of Theorem 1.1.

\section{Appendix: some technical lemmas}

We prove here some technical results used throughout the paper. The notations are introduced in Section 1.

Lemma A.1. Let $z_{i}=z\left(x_{i}\right)$ for all $i \in \mathbb{Z}$, where $z \in C(\mathbb{R})$ satisfies $\partial_{x} z \in$ $L_{l o c}^{1}(\mathbb{R})$, and $\left(x_{i}\right)$ is a uniform grid of length $\Delta x$. Then for any bounded interval $[a, b]$,

$$
T V 2_{[a, b]}\left(\left(z_{i}\right)\right) \equiv \sum_{\left[x_{i}, x_{i+1}\right] \subset[a, b]}\left(z_{i+1}-z_{i}\right)^{2}
$$

verifies

$$
T V 2_{[a, b]}\left(\left(z_{i}\right)\right) \leq\left(\int_{a}^{b}\left|\partial_{x} z(x)\right| d x\right)^{2}
$$


and

$$
T V 2_{[a, b]}\left(\left(z_{i}\right)\right) \rightarrow 0 \quad \text { as } \Delta x \rightarrow 0 .
$$

Proof. We have

$$
z_{i+1}-z_{i}=z\left(x_{i+1}\right)-z\left(x_{i}\right)=\int_{x_{i}}^{x_{i+1}} \partial_{x} z(x) d x,
$$

thus for $\left[x_{i}, x_{i+1}\right] \subset[a, b]$

$$
\left|z_{i+1}-z_{i}\right| \leq \int_{x_{i}}^{x_{i+1}}\left|\partial_{x} z(x)\right| d x \leq \int_{a}^{b}\left|\partial_{x} z(x)\right| d x .
$$

It follows that

$$
\begin{aligned}
\sum\left(z_{i+1}-z_{i}\right)^{2} & \leq \sum \int_{x_{i}}^{x_{i+1}}\left|\partial_{x} z(x)\right| d x \times \int_{a}^{b}\left|\partial_{x} z(x)\right| d x \\
& \leq\left(\int_{a}^{b}\left|\partial_{x} z(x)\right| d x\right)^{2},
\end{aligned}
$$

which proves (A.2). Next, when $z$ is Lipschitz continuous one has $\left|z_{i+1}-z_{i}\right| \leq$ $\operatorname{Lip}(z) \Delta x$, thus $T V 2_{[a, b]}\left(\left(z_{i}\right)\right) \leq \operatorname{Lip}(z)^{2} \Delta x(b-a)$ and (A.3) holds. When we have only $\partial_{x} z \in L_{l o c}^{1}$, for any $\varepsilon>0$ one can find $z_{\varepsilon} \in \operatorname{Lip}(\mathbb{R})$ such that $\left\|\partial_{x} z-\partial_{x} z_{\varepsilon}\right\|_{L^{1}([a, b])} \leq \varepsilon$, and if follows that (A.3) also holds.

Lemma A.2. Let $U_{k}=\left(h_{k}, h_{k} u_{k}\right)$ for $k=1$, 2 with $h_{k} \geq 0$. Then

$$
\begin{aligned}
& \frac{g^{2} \pi^{2}}{6}\left(2 M_{1}+M_{2}\right)\left(M_{1}-M_{2}\right)^{2} \\
= & H_{0}\left(M_{2}\right)-H_{0}\left(M_{1}\right)-\eta^{\prime}\left(U_{1}\right)\left(\begin{array}{l}
1 \\
\xi
\end{array}\right)\left(M_{2}-M_{1}\right) \\
& -\mathbb{1}_{\left(\xi-u_{1}\right)^{2}>2 g h_{1}} M_{2}\left(\frac{\left(\xi-u_{1}\right)^{2}}{2}-g h_{1}\right),
\end{aligned}
$$

where $M_{k} \equiv M_{k}(\xi) \equiv M\left(U_{k}, \xi\right)$ and $M(U, \xi)$ is defined in $(1.9), H_{0}(f) \equiv$ $H_{0}(f, \xi)$ is defined in (1.12).

Proof. This lemma indeed gives the remainder in the inequality (1.16). Using the identity

$$
b^{3}-a^{3}-3 a^{2}(b-a)=(b+2 a)(b-a)^{2},
$$

one has

$$
\frac{g^{2} \pi^{2}}{6}\left(2 M_{1}+M_{2}\right)\left(M_{1}-M_{2}\right)^{2}=H_{0}\left(M_{2}\right)-H_{0}\left(M_{1}\right)-H_{0}^{\prime}\left(M_{1}\right)\left(M_{2}-M_{1}\right),
$$


where we donote $H_{0}^{\prime}(f, \xi) \equiv \frac{\partial}{\partial f} H_{0}(f, \xi)$. Thus we have to prove that

$$
\begin{array}{r}
\quad\left(\eta^{\prime}\left(U_{1}\right)\left(\begin{array}{l}
1 \\
\xi
\end{array}\right)-H_{0}^{\prime}\left(M_{1}\right)\right)\left(M_{2}-M_{1}\right) \\
=-\mathbb{1}_{\left(\xi-u_{1}\right)^{2}>2 g h_{1}} M_{2}\left(\frac{\left(\xi-u_{1}\right)^{2}}{2}-g h_{1}\right) .
\end{array}
$$

On the one hand we compute according to (1.17)

$$
\eta^{\prime}\left(U_{1}\right)\left(\begin{array}{l}
1 \\
\xi
\end{array}\right)=g h_{1}-\frac{u_{1}^{2}}{2}+u_{1} \xi=g h_{1}+\frac{\xi^{2}}{2}-\frac{\left(\xi-u_{1}\right)^{2}}{2} .
$$

On the other hand we have using (1.9)

$$
H_{0}^{\prime}\left(M_{1}\right)=\frac{\xi^{2}}{2}+\frac{g^{2} \pi^{2}}{2} M_{1}^{2}=\frac{\xi^{2}}{2}+\left(g h_{1}-\frac{\left(\xi-u_{1}\right)^{2}}{2}\right)_{+} .
$$

Subtracting (A.12) to (A.11) it follows that

$$
\eta^{\prime}\left(U_{1}\right)\left(\begin{array}{l}
1 \\
\xi
\end{array}\right)-H_{0}^{\prime}\left(M_{1}\right)=-\mathbb{1}_{\left(\xi-u_{1}\right)^{2}>2 g h_{1}}\left(\frac{\left(\xi-u_{1}\right)^{2}}{2}-g h_{1}\right)
$$

and therefore that

$$
\begin{aligned}
& \left(\eta^{\prime}\left(U_{1}\right)\left(\begin{array}{l}
1 \\
\xi
\end{array}\right)-H_{0}^{\prime}\left(M_{1}\right)\right)\left(M_{2}-M_{1}\right) \\
= & -\mathbb{1}_{\left(\xi-u_{1}\right)^{2}>2 g h_{1}}\left(\frac{\left(\xi-u_{1}\right)^{2}}{2}-g h_{1}\right)\left(M_{2}-M_{1}\right) .
\end{aligned}
$$

Finally we notice that

$$
\left(\xi-u_{1}\right)^{2} \geq 2 g h_{1} \Longleftrightarrow M_{1}=0,
$$

thus we get (A.10), which concludes the proof.

Lemma A.3. There exists some constant $\alpha>0$, depending only on the gravity constant $g$ and on the constants $h_{m}, h_{M}, u_{M}$ involved in (1.38), such that

$$
\begin{aligned}
& \int_{\mathbb{R}}|\xi|\left(H_{0}\left(M_{2}\right)-H_{0}\left(M_{1}\right)-\eta^{\prime}\left(U_{1}\right)\left(\begin{array}{l}
1 \\
\xi
\end{array}\right)\left(M_{2}-M_{1}\right)\right) d \xi \\
& \geq \alpha\left(\eta\left(U_{2}\right)-\eta\left(U_{1}\right)-\eta^{\prime}\left(U_{1}\right)\left(U_{2}-U_{1}\right)\right)
\end{aligned}
$$

for all $U_{1}, U_{2} \in \mathcal{U}_{h_{m}, h_{M}, u_{M}}$ defined by (1.38) and where $M_{k} \equiv M_{k}(\xi) \equiv$ $M\left(U_{k}, \xi\right)$, with $M(U, \xi)$ defined in $(1.9), H_{0}(f) \equiv H_{0}(f, \xi)$ is defined in (1.12) and $\eta(U)$ is defined in (1.3). 
Proof. Note that without the factor $|\xi|$ in (A.16), the inequality would become an equality with $\alpha=1$. Thus the difficulty is to show that small values of $\xi$ do not make a significant contribution. The idea is to make a linear combination in the variable $\eta^{\prime}(U)$ as in [14, Lemma 2.3], but the difficulty is that in this variable, the set where $h \geq 0$ is not convex. This is why we have to be far from vacuum. We set

$$
\widehat{\mathcal{U}}_{m}=\left\{(h, h u) \in \mathbb{R}^{2}, h \geq h_{m}\right\},
$$

and we first deal with the case

$$
U_{1}=\left(\begin{array}{c}
h_{1} \\
h_{1} u_{1}
\end{array}\right) \text { and } U_{2}=\left(\begin{array}{c}
h_{2} \\
h_{2} u_{2}
\end{array}\right) \in \widehat{\mathcal{U}}_{m} \text {, such that }\left|u_{1}-u_{2}\right| \leq \sqrt{g h_{m}}
$$

In this case we have

$$
\forall t \in[0,1],(1-t) \eta^{\prime}\left(U_{1}\right)+t \eta^{\prime}\left(U_{2}\right) \in \eta^{\prime}\left(\widetilde{U}_{m}\right) .
$$

with

$$
\widetilde{\mathcal{U}}_{m}=\left\{(h, h u) \in \mathbb{R}^{2}, h \geq \frac{h_{m}}{2}\right\} .
$$

Indeed we notice that using (1.17),

$$
\left(\begin{array}{l}
V_{1} \\
V_{2}
\end{array}\right) \in \eta^{\prime}\left(\widetilde{\mathcal{U}}_{m}\right) \Longleftrightarrow V_{1} \geq g \frac{h_{m}}{2}-\frac{V_{2}^{2}}{2} .
$$

Thus (A.19) is equivalent to

$$
\begin{aligned}
& \forall t \in[0,1], \forall h_{1}, h_{2} \geq h_{m}, \forall u_{1}, u_{2} \in \mathbb{R}, \text { such that }\left|u_{1}-u_{2}\right| \leq \sqrt{g h_{m}} \\
& (1-t)\left(g h_{1}-\frac{u_{1}^{2}}{2}\right)+t\left(g h_{2}-\frac{u_{2}^{2}}{2}\right) \geq g \frac{h_{m}}{2}-\frac{1}{2}\left((1-t) u_{1}+t u_{2}\right)^{2} .
\end{aligned}
$$

Thus we have to check that

$$
\begin{aligned}
& \forall t \in[0,1], \forall u_{1}, u_{2} \in \mathbb{R}, \text { such that }\left|u_{1}-u_{2}\right| \leq \sqrt{g h_{m}} \\
& (1-t)\left(g h_{m}-\frac{u_{1}^{2}}{2}\right)+t\left(g h_{m}-\frac{u_{2}^{2}}{2}\right) \geq g \frac{h_{m}}{2}-\frac{1}{2}\left((1-t) u_{1}+t u_{2}\right)^{2} .
\end{aligned}
$$

This inequality simplifies to

$$
\frac{g h_{m}}{2} \geq \frac{t(1-t)}{2}\left(u_{1}-u_{2}\right)^{2},
$$


which holds true when $t \in[0,1]$ and $\left|u_{1}-u_{2}\right| \leq 2 \sqrt{g h_{m}}$. This proves (A.19).

According to the property (A.19) we can now define a path $v(t) \in \widetilde{\mathcal{U}}_{m}$, for $0 \leq t \leq 1$, connecting the two states $U_{1}, U_{2}$ satisfying (A.18), by

$$
\eta^{\prime}(v(t))=(1-t) \eta^{\prime}\left(U_{1}\right)+t \eta^{\prime}\left(U_{2}\right)
$$

Such a definition is possible because $\eta^{\prime}$ is a diffeormorphism, see (1.17). It enables us to set

$$
\begin{aligned}
\phi(t)=\int_{\mathbb{R}}|\xi|\left(H_{0}(M(v(t), \xi), \xi)-H_{0}\left(M\left(U_{1}, \xi\right), \xi\right)\right. \\
\left.-\eta^{\prime}\left(U_{1}\right)\left(\begin{array}{l}
1 \\
\xi
\end{array}\right)\left(M(v(t), \xi)-M\left(U_{1}, \xi\right)\right)\right) d \xi \\
-\alpha\left(\eta(v(t))-\eta\left(U_{1}\right)-\eta^{\prime}\left(U_{1}\right)\left(v(t)-U_{1}\right)\right) .
\end{aligned}
$$

We notice that $\phi(0)=0$, and the desired inequality (A.16) is equivalent to $\phi(1) \geq 0$. Thus it is sufficient to prove that $\phi$ is nondecreasing. Using the fact that

$$
\eta^{\prime}(U)\left(\begin{array}{l}
1 \\
\xi
\end{array}\right)=H_{0}^{\prime}(M(U, \xi), \xi), \text { for all } \xi \in \mathbb{R} \text { such that } M(U, \xi)>0
$$

we can compute

$$
\begin{aligned}
\phi^{\prime}(t)= & \int_{\mathbb{R}}|\xi|\left(\eta^{\prime}(v(t))-\eta^{\prime}\left(U_{1}\right)\right)\left(\begin{array}{l}
1 \\
\xi
\end{array}\right) M^{\prime}(v(t), \xi) v^{\prime}(t) d \xi \\
& -\alpha\left(\eta^{\prime}(v(t))-\eta^{\prime}\left(U_{1}\right)\right) v^{\prime}(t) .
\end{aligned}
$$

Moreover, using that

$$
\eta^{\prime}(v(t))-\eta^{\prime}\left(U_{1}\right)=t\left(\eta^{\prime}\left(U_{2}\right)-\eta^{\prime}\left(U_{1}\right)\right)=t \eta^{\prime \prime}(v(t)) v^{\prime}(t),
$$

we get

$$
\begin{aligned}
\phi^{\prime}(t)= & t \int_{\mathbb{R}}|\xi| \eta^{\prime \prime}(v(t)) v^{\prime}(t)\left(\begin{array}{l}
1 \\
\xi
\end{array}\right) M^{\prime}(v(t), \xi) v^{\prime}(t) d \xi \\
& -\alpha t \eta^{\prime \prime}(v(t)) v^{\prime}(t) v^{\prime}(t) .
\end{aligned}
$$

This can be rewritten as

$$
\begin{aligned}
\phi^{\prime}(t)= & t \int_{\mathbb{R}}|\xi| M^{\prime}(v(t), \xi) \otimes\left(\eta^{\prime \prime}(v(t))\left(\begin{array}{l}
1 \\
\xi
\end{array}\right)\right) \cdot v^{\prime}(t) \cdot v^{\prime}(t) d \xi \\
& -\alpha t \eta^{\prime \prime}(v(t)) \cdot v^{\prime}(t) \cdot v^{\prime}(t) .
\end{aligned}
$$


Thus now it is sufficient for getting (A.16) to prove that

$$
\begin{aligned}
& \forall U \in \widetilde{\mathcal{U}}_{m}, \forall X \in \mathbb{R}^{2} \\
& \int_{\mathbb{R}}|\xi| M^{\prime}(U, \xi) \otimes\left(\eta^{\prime \prime}(U)\left(\begin{array}{l}
1 \\
\xi
\end{array}\right)\right) \cdot X \cdot X d \xi \geq \alpha \eta^{\prime \prime}(U) \cdot X \cdot X .
\end{aligned}
$$

For all $U \in \widetilde{\mathcal{U}}_{m}$ and $\xi \in \mathbb{R}$ such that $M(U, \xi)>0$, we compute

$$
\eta^{\prime}(U)\left(\begin{array}{l}
1 \\
\xi
\end{array}\right)=H_{0}^{\prime}(M(U, \xi), \xi)
$$

and

$$
\eta^{\prime \prime}(U)\left(\begin{array}{l}
1 \\
\xi
\end{array}\right)=H_{0}^{\prime \prime}(M(U, \xi), \xi) M^{\prime}(U, \xi) .
$$

Moreover one can check that

$$
H_{0}^{\prime \prime}(M(U, \xi))=g^{2} \pi^{2} M(U, \xi) .
$$

Thus we obtain

$$
\begin{aligned}
& \int_{\mathbb{R}}|\xi| M^{\prime}(U, \xi) \otimes\left(\eta^{\prime \prime}(U)\left(\begin{array}{l}
1 \\
\xi
\end{array}\right)\right) d \xi \\
= & g^{2} \pi^{2} \int_{M(U, \xi)>0}|\xi| M(U, \xi) M^{\prime}(U, \xi) \otimes M^{\prime}(U, \xi) d \xi,
\end{aligned}
$$

and therefore the desired inequality (A.32) can be written

$$
\begin{aligned}
& \forall U \in \widetilde{\mathcal{U}}_{m}, \forall X \in \mathbb{R}^{2} \\
& g^{2} \pi^{2} \int_{M(U, \xi)>0}|\xi| M(U, \xi)\left(M^{\prime}(U, \xi) X\right)^{2} d \xi \geq \alpha \eta^{\prime \prime}(U) \cdot X \cdot X .
\end{aligned}
$$

According to (1.17), we have

$$
\begin{gathered}
\eta^{\prime}(h, q)=\left(-\frac{1}{2} \frac{q^{2}}{h^{2}}+g h, \frac{q}{h}\right), \\
\eta^{\prime \prime}(h, q)=\left(\begin{array}{cc}
q^{2} \\
h^{3}+g & -\frac{q}{h^{2}} \\
-\frac{q}{h^{2}} & \frac{1}{h}
\end{array}\right)=\left(\begin{array}{cc}
\frac{u^{2}}{h}+g & -\frac{u}{h} \\
-\frac{u}{h} & \frac{1}{h}
\end{array}\right) .
\end{gathered}
$$

Denoting $X=\left(\begin{array}{l}x_{1} \\ x_{2}\end{array}\right)$, we get

$$
\begin{aligned}
& \eta^{\prime \prime}(U) \cdot X \cdot X \\
= & \left(g+\frac{u^{2}}{h}\right) x_{1}^{2}+\frac{1}{h} x_{2}^{2}-\frac{2 u}{h} x_{1} x_{2} \\
= & g x_{1}^{2}+\frac{1}{h}\left(x_{2}-u x_{1}\right)^{2} \\
= & g x_{1}^{2}+h x_{3}^{2},
\end{aligned}
$$


where we denote

$$
x_{3}=\frac{1}{h}\left(x_{2}-u x_{1}\right) .
$$

In order to compute $M^{\prime}(U, \xi) X$, with (1.9) we compute the partial derivatives where $M(U, \xi)>0$,

$$
\begin{gathered}
\partial_{h} M(U, \xi)=\frac{1}{2 g \pi}\left(2 g h-(\xi-u)^{2}\right)^{-1 / 2}\left(2 g-2 \frac{u}{h}(\xi-u)\right), \\
\partial_{h u} M(U, \xi)=\frac{1}{2 g \pi}\left(2 g h-(\xi-u)^{2}\right)^{-1 / 2} \frac{2}{h}(\xi-u) .
\end{gathered}
$$

It leads to the formula (where $M(U, \xi)>0$ )

$$
\begin{aligned}
M^{\prime}(U, \xi) X & =\frac{M(U, \xi)^{-1}}{g^{2} \pi^{2}}\left(g x_{1}+\frac{(\xi-u)}{h}\left(x_{2}-u x_{1}\right)\right) \\
& =\frac{M(U, \xi)^{-1}}{g^{2} \pi^{2}}\left(g x_{1}+(\xi-u) x_{3}\right) .
\end{aligned}
$$

Using (A.44) in the integral of (A.37) we get

$$
\begin{aligned}
& g^{2} \pi^{2} \int_{M(U, \xi)>0}|\xi| M(U, \xi)\left(M^{\prime}(U, \xi) X\right)^{2} d \xi \\
= & \frac{1}{g^{2} \pi^{2}} \int_{M(U, \xi)>0}|\xi| \frac{1}{M(U, \xi)}\left(g x_{1}+(\xi-u) x_{3}\right)^{2} d \xi \\
= & \frac{1}{g \pi} \int_{(\xi-u)^{2}<2 g h}|\xi| \frac{1}{\left(2 g h-(\xi-u)^{2}\right)^{1 / 2}}\left(g x_{1}+(\xi-u) x_{3}\right)^{2} d \xi:=I .
\end{aligned}
$$

As for (A.16), we notice that without the factor $|\xi|$, the inequality (A.37) would become an equality with $\alpha=1$ (compute the integral (A.45) without the factor $|\xi|$ ). With the factor $|\xi|$, we use the substitution $v=\xi-u$ in (A.45) and the convention that if $u=0$ then $\operatorname{sgn}(u)=1$, to obtain

$$
\begin{aligned}
I & \geq \frac{1}{g \pi \sqrt{2 g h}} \int_{|v|<\sqrt{2 g h}}|v+u|\left(g x_{1}+v x_{3}\right)^{2} d v \\
& \geq \frac{1}{g \pi \sqrt{2 g h}} \int_{|v|<\sqrt{2 g h}, \operatorname{sgn}(v)=\operatorname{sgn}(u)}(|v|+|u|)\left(g x_{1}+|v| \operatorname{sgn}(u) x_{3}\right)^{2} d v \\
& \geq \frac{1}{g \pi \sqrt{2 g h}} \int_{0}^{\sqrt{2 g h}} v\left(g x_{1}+v \operatorname{sgn}(u) x_{3}\right)^{2} d v \\
& \geq \frac{1}{2 g \pi} \int_{\frac{\sqrt{2 g h}}{2}}^{\sqrt{2 g h}}\left(g x_{1}+v \operatorname{sgn}(u) x_{3}\right)^{2} d v \\
& =\frac{\sqrt{h}}{\sqrt{2 g} \pi} \int_{1 / 2}^{1}\left(g x_{1}+\xi \sqrt{2 g h} \operatorname{sgn}(u) x_{3}\right)^{2} d \xi
\end{aligned}
$$


The last integral is a positive definite quadratic form with respect to $y_{1}=g x_{1}$ and $y_{3}=\sqrt{2 g h} \operatorname{sgn}(u) x_{3}$. Thus we have for some absolute constant $C_{0}>0$ (one can check that $C_{0}=1 /(8 \times 13)$ works)

$$
I \geq C_{0} \frac{\sqrt{h}}{\sqrt{2 g} \pi}\left(\left(g x_{1}\right)^{2}+g h x_{3}^{2}\right)=C_{0} \frac{\sqrt{g h}}{\sqrt{2} \pi}\left(g x_{1}^{2}+h x_{3}^{2}\right) .
$$

Therefore by (A.45), (A.47) we get

$$
g^{2} \pi^{2} \int_{M(U, \xi)>0}|\xi| M(U, \xi)\left(M^{\prime}(U, \xi) X\right)^{2} d \xi \geq C_{0} \frac{\sqrt{g h}}{\sqrt{2} \pi}\left(g x_{1}^{2}+h x_{3}^{2}\right) .
$$

Because of (A.40), this proves that (A.37) holds with $\alpha_{1}=C_{0} \sqrt{g h_{m}} / 2 \pi$. We conclude that (A.16) holds for all $U_{1}, U_{2} \in \widehat{\mathcal{U}}_{m}$ such that $\left|u_{1}-u_{2}\right| \leq \sqrt{g h_{m}}$, with the constant $\alpha_{1}$.

Thus, to conclude the lemma it is now sufficient to prove that

$$
\begin{aligned}
& \exists \alpha_{2}>0, \quad \forall U_{1}, U_{2} \in \mathcal{U}_{h_{m}, h_{M}, u_{M}} \text { such that }\left|u_{1}-u_{2}\right|>\sqrt{g h_{m}}, \\
& \int_{\mathbb{R}}|\xi|\left(H_{0}\left(M_{2}\right)-H_{0}\left(M_{1}\right)-\eta^{\prime}\left(U_{1}\right)\left(\begin{array}{l}
1 \\
\xi
\end{array}\right)\left(M_{2}-M_{1}\right)\right) d \xi \geq \alpha_{2} .
\end{aligned}
$$

Indeed, when $U_{1}, U_{2} \in \mathcal{U}_{h_{m}, h_{M}, u_{M}}$ we have

$\eta\left(U_{2}\right)-\eta\left(U_{1}\right)-\eta^{\prime}\left(U_{1}\right)\left(U_{2}-U_{1}\right)=g \frac{\left(h_{2}-h_{1}\right)^{2}}{2}+h_{2} \frac{\left(u_{2}-u_{1}\right)^{2}}{2} \leq C\left(g, h_{M}, u_{M}\right)$,

thus when (A.49) holds, we deduce (A.16). Proceeding by reductio ad absurdum, let us assume that (A.49) does not hold. Thus

$$
\begin{aligned}
& \forall n>0, \quad \exists U_{1}^{n}, U_{2}^{n} \in \mathcal{U}_{h_{m}, h_{M}, u_{M}}, \text { such that } \\
& \left|u_{1}^{n}-u_{2}^{n}\right|>\sqrt{g h_{m}} \\
& \text { and } \int_{\mathbb{R}}|\xi|\left(H_{0}\left(M_{2}^{n}\right)-H_{0}\left(M_{1}^{n}\right)-\eta^{\prime}\left(U_{1}^{n}\right)\left(\begin{array}{l}
1 \\
\xi
\end{array}\right)\left(M_{2}^{n}-M_{1}^{n}\right)\right) d \xi \leq \frac{1}{n},
\end{aligned}
$$

where $M_{i}^{n}=M\left(U_{i}^{n}, \xi\right)$. As $\mathcal{U}_{h_{m}, h_{M}, u_{M}}$ is a closed and bounded set, we can extract a subsequence (that we still denote $U_{1}^{n}, U_{2}^{n}$ ) such that

$$
U_{1}^{n} \rightarrow U_{1} \in \mathcal{U}_{h_{m}, h_{M}, u_{M}}, \quad U_{2}^{n} \rightarrow U_{2} \in \mathcal{U}_{h_{m}, h_{M}, u_{M}}
$$

with

$$
\left|u_{1}-u_{2}\right| \geq \sqrt{g h_{m}}
$$


By Lebesgue'theorem, (A.51) implies that

$$
\int_{\mathbb{R}}|\xi|\left(H_{0}\left(M_{2}\right)-H_{0}\left(M_{1}\right)-\eta^{\prime}\left(U_{1}\right)\left(\begin{array}{l}
1 \\
\xi
\end{array}\right)\left(M_{2}-M_{1}\right)\right) d \xi \leq 0 .
$$

We also know by (A.7) that

$$
H_{0}\left(M_{2}\right)-H_{0}\left(M_{1}\right)-\eta^{\prime}\left(U_{1}\right)\left(\begin{array}{l}
1 \\
\xi
\end{array}\right)\left(M_{2}-M_{1}\right) \geq \frac{g^{2} \pi^{2}}{6}\left(2 M_{1}+M_{2}\right)\left(M_{1}-M_{2}\right)^{2}
$$

and therefore we get that

$$
\left(2 M_{1}+M_{2}\right)\left(M_{1}-M_{2}\right)^{2}=0 \text { for almost every } \xi .
$$

This implies that $M_{1}=M_{2}$ a.e. and therefore that $U_{1}=U_{2}$, in contradiction with (A.53). This concludes the proof of Lemma A.3.

Lemma A.4. One has

$$
\begin{aligned}
& \int_{\left(\xi-u_{1}\right)^{2}>2 g h_{1}}|\xi| M\left(U_{2}, \xi\right)\left(\frac{\left(\xi-u_{1}\right)^{2}}{2}-g h_{1}\right) d \xi \\
\leq & \frac{4\left(\left|u_{2}\right|+\sqrt{2 g h_{2}}\right)}{g \pi \sqrt{g h_{2}}} K^{\frac{3}{2}} \min \left(g h_{2}, K\right),
\end{aligned}
$$

for all $U_{1}=\left(h_{1}, h_{1} u_{1}\right), h_{1}>0$ and $U_{2}=\left(h_{2}, h_{2} u_{2}\right), h_{2}>0$, where $M(U, \xi)$ is defined in (1.9), and

$$
K=g\left|h_{1}-h_{2}\right|+\left(\left|u_{2}\right|+\sqrt{2 g h_{2}}\right)\left|u_{1}-u_{2}\right|+\frac{1}{2}\left|u_{1}^{2}-u_{2}^{2}\right| .
$$

Proof. We notice that for all $\xi \in \operatorname{supp}\left(M_{2}\right)$ one has $|\xi| \leq\left|u_{2}\right|+\sqrt{2 g h_{2}}$, thus we have

$$
\begin{aligned}
& \left|g h_{2}-\frac{\left(\xi-u_{2}\right)^{2}}{2}-\left(g h_{1}-\frac{\left(\xi-u_{1}\right)^{2}}{2}\right)\right| \\
= & \left|g\left(h_{2}-h_{1}\right)+\xi\left(u_{2}-u_{1}\right)-\frac{1}{2}\left(u_{2}^{2}-u_{1}^{2}\right)\right| \\
\leq & K .
\end{aligned}
$$

Therefore, using that $\xi \in \operatorname{supp}\left(M_{1}\right)^{c} \cap \operatorname{supp}\left(M_{2}\right)$ iff $g h_{2}-\frac{\left(\xi-u_{2}\right)^{2}}{2} \geq 0$ and $\frac{\left(\xi-u_{1}\right)^{2}}{2}-g h_{1}>0$, we get

$$
\left|g h_{2}-\frac{\left(\xi-u_{2}\right)^{2}}{2}\right|+\left|\frac{\left(\xi-u_{1}\right)^{2}}{2}-g h_{1}\right| \leq K
$$


Using (1.9) and (A.60), we get

$$
\begin{aligned}
& \int_{\left(\xi-u_{1}\right)^{2}>2 g h_{1}}|\xi| M\left(U_{2}, \xi\right)\left(\frac{\left(\xi-u_{1}\right)^{2}}{2}-g h_{1}\right) d \xi \\
= & \frac{\sqrt{2}}{g \pi} \int_{\operatorname{Supp}\left(M_{1}\right)^{c} \cap \operatorname{supp}\left(M_{2}\right)}|\xi|\left(g h_{2}-\frac{\left(\xi-u_{2}\right)^{2}}{2}\right)^{1 / 2}\left(\frac{\left(\xi-u_{1}\right)^{2}}{2}-g h_{1}\right) d \xi \\
\leq & \frac{\sqrt{2}}{g \pi}\left(\left|u_{2}\right|+\sqrt{2 g h_{2}}\right)\left|\operatorname{supp}\left(M_{1}\right)^{c} \cap \operatorname{supp}\left(M_{2}\right)\right| K^{3 / 2}
\end{aligned}
$$

Thus it is now sufficient for getting (A.57) to prove that

$$
\left|\operatorname{supp}\left(M_{1}\right)^{c} \cap \operatorname{supp}\left(M_{2}\right)\right| \leq \frac{4 \min \left(g h_{2}, K\right)}{\sqrt{2 g h_{2}}} .
$$

We observe from (A.60) that for $\xi \in \operatorname{supp}\left(M_{1}\right)^{c} \cap \operatorname{supp}\left(M_{2}\right)$ one has

$$
P(\xi) \leq 0
$$

where

$$
P(\xi) \equiv g h_{2}-\frac{\left(\xi-u_{2}\right)^{2}}{2}-K
$$

We notice that when $\xi=u_{2}, P$ reaches a maximum equals to $g h_{2}-K$, and we distinguish two cases:

- If $K \geq g h_{2}$ then

$$
\left|\operatorname{supp}\left(M_{1}\right)^{c} \cap \operatorname{supp}\left(M_{2}\right)\right| \leq\left|\operatorname{supp}\left(M_{2}\right)\right|=2 \sqrt{2 g h_{2}},
$$

which concludes (A.62).

- If $K<g h_{2}$, then the maximum of $P$ is positive and using (A.63) we get that for $\xi \in \operatorname{supp}\left(M_{1}\right)^{c} \cap \operatorname{supp}\left(M_{2}\right)$ we have

$$
\xi \in\left[u_{2}-\sqrt{2 g h_{2}}, r_{1}\right] \bigcup\left[r_{2}, u_{2}+\sqrt{2 g h_{2}}\right],
$$

with $r_{1}<u_{2}<r_{2}$ are such that $P\left(r_{1}\right)=P\left(r_{2}\right)=0$. We have $u_{2}-$ $\sqrt{2 g h_{2}}<r_{1}$ because $P\left(u_{2}-\sqrt{2 g h_{2}}\right)=-K<0$, and $r_{2}<u_{2}+\sqrt{2 g h_{2}}$ because $P\left(u_{2}+\sqrt{2 g h_{2}}\right)=-K<0$. This configuration is illustrated on the following picture. 


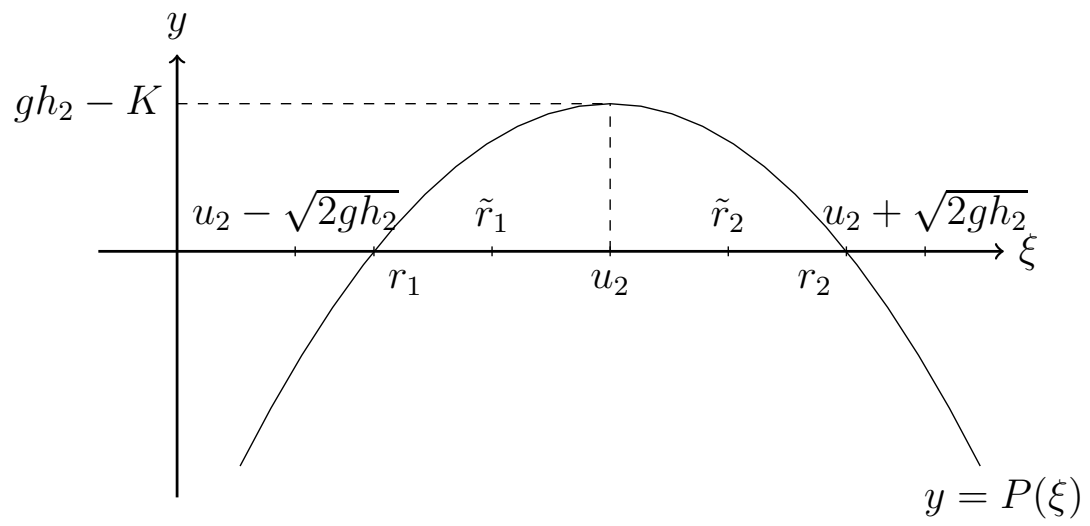

Graph of $\xi \mapsto P(\xi)$ when $K<g h_{2}$

Thus

$\left|\operatorname{supp}\left(M_{1}\right)^{c} \cap \operatorname{supp}\left(M_{2}\right)\right| \leq\left|r_{1}-\left(u_{2}-\sqrt{2 g h_{2}}\right)\right|+\left|u_{2}+\sqrt{2 g h_{2}}-r_{2}\right|$.

We set

$$
\tilde{r}_{1}=u_{2}-\sqrt{2 g h_{2}}+\frac{2 K}{\sqrt{2 g h_{2}}}
$$

and we notice that

$$
\frac{2 K}{\sqrt{2 g h_{2}}}<\sqrt{2 g h_{2}}
$$

because of the assumption $K<g h_{2}$. Thus we obtain that

$$
\tilde{r}_{1}<u_{2}
$$

Moreover

$$
\begin{aligned}
P\left(\tilde{r}_{1}\right) & =g h_{2}-\frac{\left(\tilde{r}_{1}-u_{2}\right)^{2}}{2}-K \\
& =g h_{2}-\frac{1}{2}\left(-\sqrt{2 g h_{2}}+\frac{2 K}{\sqrt{2 g h_{2}}}\right)^{2}-K \\
& =-\frac{K^{2}}{g h_{2}}+2 K-K=K\left(1-\frac{K}{g h_{2}}\right)>0 .
\end{aligned}
$$

With (A.70) we deduce that

$$
r_{1}<\tilde{r}_{1}<u_{2}
$$


Similarly we set

$$
\tilde{r}_{2}=u_{2}+\sqrt{2 g h_{2}}-\frac{2 K}{\sqrt{2 g h_{2}}}
$$

and by the same arguments we obtain that

$$
u_{2}<\tilde{r}_{2}<r_{2} .
$$

Putting together (A.72) and (A.74), we get

$$
\begin{aligned}
& \left|r_{1}-\left(u_{2}-\sqrt{2 g h_{2}}\right)\right|+\left|u_{2}+\sqrt{2 g h_{2}}-r_{2}\right| \\
\leq & \left|\tilde{r}_{1}-\left(u_{2}-\sqrt{2 g h_{2}}\right)\right|+\left|u_{2}+\sqrt{2 g h_{2}}-\tilde{r}_{2}\right| \\
= & \frac{4 K}{\sqrt{2 g h_{2}}} .
\end{aligned}
$$

With (A.67) we get (A.62) in the case $K<g h_{2}$, and this concludes the proof of Lemma A.4.

Lemma A.5. There exists some $C>0$ depending only on $g, h_{m}, h_{M}, u_{M}$ such that

$$
\begin{aligned}
& \int_{\mathbb{R}}|\xi| \frac{g^{2} \pi^{2}}{6}\left(2 M_{1}+M_{2}\right)\left(M_{1}-M_{2}\right)^{2} d \xi \\
\geq & C\left(g \frac{\left(h_{2}-h_{1}\right)^{2}}{2}+h_{m} \frac{\left(u_{2}-u_{1}\right)^{2}}{2}\right),
\end{aligned}
$$

for all $U_{1}, U_{2} \in \mathcal{U}_{h_{m}, h_{M}, u_{M}}$ defined by (1.38) and where $M_{k} \equiv M_{k}(\xi) \equiv$ $M\left(U_{k}, \xi\right)$, with $M(U, \xi)$ defined by $(1.9)$.

Proof. Let $U_{1}, U_{2} \in \mathcal{U}_{h_{m}, h_{M}, u_{M}}$. According to Lemma A.2 we have

$$
\begin{aligned}
& \int_{\mathbb{R}}|\xi| \frac{g^{2} \pi^{2}}{6}\left(2 M_{1}+M_{2}\right)\left(M_{1}-M_{2}\right)^{2} d \xi \\
= & \int_{\mathbb{R}}|\xi|\left(H_{0}\left(M_{2}\right)-H_{0}\left(M_{1}\right)-\eta^{\prime}\left(U_{1}\right)\left(\begin{array}{l}
1 \\
\xi
\end{array}\right)\left(M_{2}-M_{1}\right)\right) d \xi \\
& -\int_{\left(\xi-u_{1}\right)^{2}>2 g h_{1}}|\xi| M_{2}\left(\frac{\left(\xi-u_{1}\right)^{2}}{2}-g h_{1}\right) d \xi .
\end{aligned}
$$

Let us first consider the case of data

$$
U_{1}, U_{2} \text { such that }\left|h_{1}-h_{2}\right| \leq \frac{1}{4 \widetilde{C}_{1}^{2}} \text { and }\left|u_{1}-u_{2}\right| \leq \frac{1}{4 \widetilde{C}_{2}^{2}}
$$


for some positive constants $\widetilde{C}_{1}, \widetilde{C}_{2}$ depending on $g, h_{m}, h_{M}, u_{M}$ such that $4 \widetilde{C}_{2}^{2} \geq \frac{1}{\sqrt{g h_{m}}}$. These constants will be chosen further on.

For data satisfying (A.78), we are going to estimate the right-hand side of (A.77). On the one hand, in order to estimate the first term in the RHS of (A.77), we apply Lemma A.3. Since $4 \widetilde{C}_{2}^{2} \geq \frac{1}{\sqrt{g h_{m}}}$ we are in the case (A.18) and we get

$$
\begin{aligned}
& \int_{\mathbb{R}}|\xi|\left(H_{0}\left(M_{2}\right)-H_{0}\left(M_{1}\right)-\eta^{\prime}\left(U_{1}\right)\left(\begin{array}{l}
1 \\
\xi
\end{array}\right)\left(M_{2}-M_{1}\right)\right) d \xi \\
\geq & \alpha_{1}\left(\eta\left(U_{2}\right)-\eta\left(U_{1}\right)-\eta^{\prime}\left(U_{1}\right)\left(U_{2}-U_{1}\right)\right) \\
= & \alpha_{1}\left(g \frac{\left(h_{2}-h_{1}\right)^{2}}{2}+h_{2} \frac{\left(u_{2}-u_{1}\right)^{2}}{2}\right) \\
\geq & \alpha_{1}\left(g \frac{\left(h_{2}-h_{1}\right)^{2}}{2}+h_{m} \frac{\left(u_{2}-u_{1}\right)^{2}}{2}\right),
\end{aligned}
$$

with $\alpha_{1}=C_{0} \sqrt{g h_{m}} / 2 \pi$ and $C_{0}>0$ is and absolute constant. On the other hand, in order to estimate the second term in the RHS of (A.77), we apply Lemma A.4 and obtain

$$
\begin{aligned}
& \int_{\left(\xi-u_{1}\right)^{2}>2 g h_{1}}|\xi| M\left(U_{2}, \xi\right)\left(\frac{\left(\xi-u_{1}\right)^{2}}{2}-g h_{1}\right) d \xi \\
\leq & \frac{4\left(\left|u_{2}\right|+\sqrt{2 g h_{2}}\right)}{g \pi \sqrt{g h_{2}}}\left(g\left|h_{1}-h_{2}\right|+\left(\left|u_{2}\right|+\sqrt{2 g h_{2}}\right)\left|u_{1}-u_{2}\right|+\frac{1}{2}\left|u_{1}^{2}-u_{2}^{2}\right|\right)^{\frac{5}{2}} \\
\leq & C_{1}\left(g, h_{m}, h_{M}, u_{M}\right)\left(g\left|h_{1}-h_{2}\right|+C_{2}\left(g, h_{m}, h_{M}, u_{M}\right)\left|u_{1}-u_{2}\right|\right)^{\frac{5}{2}}, \quad(\mathrm{~A} .80)
\end{aligned}
$$

with

$$
\begin{array}{r}
C_{1}\left(g, h_{m}, h_{M}, u_{M}\right)=\frac{4\left(u_{M}+\sqrt{2 g h_{M}}\right)}{g \pi \sqrt{g h_{m}}}, \\
C_{2}\left(g, h_{m}, h_{M}, u_{M}\right)=2 u_{M}+\sqrt{2 g h_{M}} .
\end{array}
$$

Using the Jensen inequality we have for $a, b \geq 0$,

$$
(a+b)^{5 / 2}=2^{5 / 2}\left(\frac{a+b}{2}\right)^{5 / 2} \leq 2^{5 / 2} \frac{a^{5 / 2}+b^{5 / 2}}{2},
$$

we get

$$
\begin{aligned}
& \int_{\left(\xi-u_{1}\right)^{2}>2 g h_{1}}|\xi| M\left(U_{2}, \xi\right)\left(\frac{\left(\xi-u_{1}\right)^{2}}{2}-g h_{1}\right) d \xi \\
\leq & 2^{3 / 2} C_{1}\left(g, h_{m}, h_{M}, u_{M}\right)\left(g^{\frac{5}{2}}\left|h_{1}-h_{2}\right|^{\frac{5}{2}}+C_{2}\left(g, h_{m}, h_{M}, u_{M}\right)^{\frac{5}{2}}\left|u_{1}-u_{2}\right|^{\frac{5}{2}}\right) .
\end{aligned}
$$


Thus, using the estimates (A.79) and (A.84) in the RHS of (A.77), we get

$$
\begin{aligned}
& \int_{\mathbb{R}}|\xi| \frac{g^{2} \pi^{2}}{6}\left(2 M_{1}+M_{2}\right)\left(M_{1}-M_{2}\right)^{2} d \xi \\
\geq & \alpha_{1}\left(g \frac{\left(h_{2}-h_{1}\right)^{2}}{2}+h_{m} \frac{\left(u_{2}-u_{1}\right)^{2}}{2}\right) \\
& -2^{3 / 2} C_{1}\left(g^{\frac{5}{2}}\left|h_{1}-h_{2}\right|^{\frac{5}{2}}+C_{2}^{\frac{5}{2}}\left|u_{1}-u_{2}\right|^{\frac{5}{2}}\right) \\
= & \alpha_{1} \frac{g\left(h_{2}-h_{1}\right)^{2}}{2}\left(1-\widetilde{C}_{1}\left|h_{1}-h_{2}\right|^{\frac{1}{2}}\right) \\
& +\alpha_{1} \frac{h_{m}\left(u_{2}-u_{1}\right)^{2}}{2}\left(1-\widetilde{C}_{2}\left|u_{1}-u_{2}\right|^{\frac{1}{2}}\right),
\end{aligned}
$$

with

$$
\widetilde{C}_{1}=\frac{2^{3 / 2+1} C_{1} g^{\frac{5}{2}}}{\alpha_{1} g}, \quad \widetilde{C}_{2}=\frac{2^{3 / 2+1} C_{1} C_{2}^{\frac{5}{2}}}{\alpha_{1} h_{m}} .
$$

One can check that $\widetilde{C}_{2}>\left(g h_{m}\right)^{-1 / 4} / 2$. From (A.85), since we are dealing with $U_{1}, U_{2}$ satisfying (A.78), we get

$$
\begin{aligned}
& \int_{\mathbb{R}}|\xi| \frac{g^{2} \pi^{2}}{6}\left(2 M_{1}+M_{2}\right)\left(M_{1}-M_{2}\right)^{2} d \xi \\
\geq & \frac{\alpha_{1}}{2}\left(g \frac{\left(h_{2}-h_{1}\right)^{2}}{2}+h_{m} \frac{\left(u_{2}-u_{1}\right)^{2}}{2}\right) .
\end{aligned}
$$

At this point we have the result (A.76) for all $U_{1}, U_{2} \in \mathcal{U}_{h_{m}, h_{M}, u_{M}}$ satisfying (A.78). Thus, since the right-hand side of (A.76) is bounded, it is now sufficient to prove that

$$
\begin{aligned}
& \exists \alpha_{3}>0, \quad \forall U_{1}, U_{2} \in \mathcal{U}_{h_{m}, h_{M}, u_{M}} \text { such that } \\
& \left|h_{1}-h_{2}\right|>\frac{1}{4 \widetilde{C}_{1}^{2}} \text { or }\left|u_{1}-u_{2}\right|>\frac{1}{4 \widetilde{C}_{2}^{2}}, \\
& \text { we have } \int_{\mathbb{R}}|\xi| \frac{g^{2} \pi^{2}}{6}\left(2 M_{1}+M_{2}\right)\left(M_{1}-M_{2}\right)^{2} d \xi \geq \alpha_{3} .
\end{aligned}
$$

Using a reductio ad absurdum as in the proof of Lemma A.3, we suppose that (A.88) does not hold. Thus

$$
\begin{aligned}
& \forall n>0, \quad \exists U_{1}^{n}, U_{2}^{n} \in \mathcal{U}_{h_{m}, h_{M}, u_{M}}, \text { such that } \\
& 4 \widetilde{C}_{1}^{2}\left|h_{1}^{n}-h_{2}^{n}\right|+4 \widetilde{C}_{2}^{2}\left|u_{1}^{n}-u_{2}^{n}\right|>1 \\
& \text { and } \int_{\mathbb{R}}|\xi| \frac{g^{2} \pi^{2}}{6}\left(2 M_{1}^{n}+M_{2}^{n}\right)\left(M_{1}^{n}-M_{2}^{n}\right)^{2} d \xi \leq \frac{1}{n},
\end{aligned}
$$


where $M_{k}^{n}=M\left(U_{k}^{n}, \xi\right)$. As $\mathcal{U}_{h_{m}, h_{M}, u_{M}}$ is a closed and bounded set, we can extract a subsequence such that

$$
U_{1}^{n} \rightarrow U_{1} \in \mathcal{U}_{h_{m}, h_{M}, u_{M}}, \quad U_{2}^{n} \rightarrow U_{2} \in \mathcal{U}_{h_{m}, h_{M}, u_{M}}
$$

with

$$
4 \widetilde{C}_{1}^{2}\left|h_{1}-h_{2}\right|+4 \widetilde{C}_{2}^{2}\left|u_{1}-u_{2}\right| \geq 1,
$$

and by Lebesgue's theorem

$$
\int_{\mathbb{R}}|\xi| \frac{g^{2} \pi^{2}}{6}\left(2 M_{1}+M_{2}\right)\left(M_{1}-M_{2}\right)^{2} d \xi=0 .
$$

Therefore we get

$$
\left(2 M_{1}+M_{2}\right)\left(M_{1}-M_{2}\right)^{2}=0 \text { for almost all } \xi,
$$

itself implying that $M_{1}=M_{2}$ a.e. and therefore $U_{1}=U_{2}$, in contradiction with (A.91). This concludes the proof of Lemma A.5.

Lemma A.6. Let $U_{k}=\left(h_{k}, h_{k} u_{k}\right), k=1,2$ with $h_{k} \geq 0$. Then

$$
\begin{aligned}
& \int_{\mathbb{R}}\left|M\left(U_{1}, \xi\right)-M\left(U_{2}, \xi\right)\right| d \xi \\
& \leq \frac{2 \sqrt{3}}{\sqrt{g}}\left(g\left(h_{2}-h_{1}\right)^{2}+\min \left(h_{1}, h_{2}\right)\left(u_{2}-u_{1}\right)^{2}\right)^{\frac{1}{2}},
\end{aligned}
$$

with $M(U, \xi)$ defined by (1.9).

Proof. Let us recall that from [5, Lemma 3.11] one has

$$
\begin{array}{r}
\int_{\mathbb{R}} M\left(U_{1}, \xi\right)\left(M\left(U_{1}, \xi\right)-M\left(U_{2}, \xi\right)\right)^{2} d \xi \\
\leq \frac{3}{g^{2} \pi^{2}}\left(g\left(h_{2}-h_{1}\right)^{2}+\min \left(h_{1}, h_{2}\right)\left(u_{2}-u_{1}\right)^{2}\right) .
\end{array}
$$

Then using the Cauchy-Schwarz inequality,

$$
\begin{aligned}
& \int_{\mathbb{R}}\left|M\left(U_{1}, \xi\right)-M\left(U_{2}, \xi\right)\right| d \xi \\
\leq & \int_{M_{1}>0}\left|M_{1}-M_{2}\right| d \xi+\int_{M_{2}>0}\left|M_{1}-M_{2}\right| d \xi \\
\leq & \left(\int_{M_{1}>0} \frac{1}{M_{1}} d \xi\right)^{1 / 2}\left(\int_{M_{1}>0} M_{1}\left(M_{1}-M_{2}\right)^{2} d \xi\right)^{1 / 2} \\
& +\left(\int_{M_{2}>0} \frac{1}{M_{2}} d \xi\right)^{1 / 2}\left(\int_{M_{2}>0} M_{2}\left(M_{1}-M_{2}\right)^{2} d \xi\right)^{1 / 2}
\end{aligned}
$$


Using the substitution $v=\frac{\xi-u}{\sqrt{2 g h}}$ we get

$$
\begin{aligned}
& \int_{M(U, \xi)>0} \frac{1}{M(U, \xi)} d \xi=\int_{u-\sqrt{2 g h}}^{u+\sqrt{2 g h}} \frac{g \pi}{\left(2 g h-(\xi-u)^{2}\right)^{1 / 2}} d \xi \\
= & \int_{-1}^{1} \frac{g \pi \sqrt{2 g h}}{\sqrt{2 g h}\left(1-v^{2}\right)^{1 / 2}} d v=g \pi[\arcsin (v)]_{-1}^{1}=g \pi^{2} .
\end{aligned}
$$

Now from (A.96), using (A.95) and (A.97) we get (A.94), which concludes the proof.

Lemma A.7. Let $U_{k}=\left(h_{k}, h_{k} u_{k}\right), k=1,2$ with $h_{k} \geq 0$, and set

$$
C_{4}=\max _{v \in\left\{\left|u_{1}\right|+\sqrt{2 g h_{1}},\left|u_{2}\right|+\sqrt{2 g h_{2}}\right\}}|v|\left(1+\nu^{2} v^{2}\right)^{\frac{1}{2}}
$$

for some given $\nu>0$. Then one has denoting $\left\|\left(x_{1}, x_{2}\right)\right\|^{2}=x_{1}^{2}+\nu^{2} x_{2}^{2}$,

$$
\begin{gathered}
\left\|F\left(U_{1}\right)-F\left(U_{2}\right)\right\| \leq \frac{2 \sqrt{3}}{\sqrt{g}} C_{4}\left(g\left(h_{2}-h_{1}\right)^{2}+\min \left(h_{1}, h_{2}\right)\left(u_{2}-u_{1}\right)^{2}\right)^{\frac{1}{2}} \\
\left\|F^{+}\left(U_{1}\right)-F^{+}\left(U_{2}\right)\right\| \mid \leq \frac{2 \sqrt{3}}{\sqrt{g}} C_{4}\left(g\left(h_{2}-h_{1}\right)^{2}+\min \left(h_{1}, h_{2}\right)\left(u_{2}-u_{1}\right)^{2}\right)^{\frac{1}{2}}, \\
\left\|F^{-}\left(U_{1}\right)-F^{-}\left(U_{2}\right)\right\| \leq \frac{2 \sqrt{3}}{\sqrt{g}} C_{4}\left(g\left(h_{2}-h_{1}\right)^{2}+\min \left(h_{1}, h_{2}\right)\left(u_{2}-u_{1}\right)^{2}\right)^{\frac{1}{2}} .
\end{gathered}
$$

Proof. We recall that from (1.28),

$$
\begin{aligned}
& F^{+}(U)=\int_{\mathbb{R}} \xi \mathbb{1}_{\xi>0}\left(\begin{array}{l}
1 \\
\xi
\end{array}\right) M(U, \xi) d \xi \\
& F^{-}(U)=\int_{\mathbb{R}} \xi \mathbb{1}_{\xi<0}\left(\begin{array}{l}
1 \\
\xi
\end{array}\right) M(U, \xi) d \xi \\
& \text { and } F(U)=F^{+}(U)+F^{-}(U)=\int_{\mathbb{R}} \xi\left(\begin{array}{l}
1 \\
\xi
\end{array}\right) M(U, \xi) d \xi
\end{aligned}
$$

Thus the result is an immediate consequence of Lemma A.6 and of the fact that

$$
\forall \xi \in \operatorname{supp} M_{1} \cup \operatorname{supp} M_{2}, \quad\left\|\xi\left(\begin{array}{l}
1 \\
\xi
\end{array}\right)\right\| \leq C_{4} .
$$


Lemma A.8. With the assumptions of Theorem 1.1, let $U_{\Delta}$ be the approximate solution (1.33) and $\phi \in \mathcal{D}(\mathbb{R})$. Then there exists some $C>0$ depending only on the available bounds and on $\phi$ such that

$$
\forall t \in[0, T], \quad<\partial_{t} U_{\Delta}(t, \cdot), \phi>\leq C .
$$

Proof. Using (2.43) we get for any $t_{n} \leq t<t_{n+1}$

$$
<\partial_{t} U_{\Delta}, \phi>=A+B
$$

with

$$
A=\sum_{i} \frac{1}{\Delta t}\left[\frac{U_{i+1}^{n+1}-U_{i}^{n+1}-U_{i+1}^{n}+U_{i}^{n}}{\Delta x}\right] \int_{x_{i}}^{x_{i+1}}\left(x-x_{i}\right) \phi(x) d x
$$

and

$$
B=\sum_{i} \frac{1}{\Delta t}\left[U_{i}^{n+1}-U_{i}^{n}\right] \int_{x_{i}}^{x_{i+1}} \phi(x) d x .
$$

First we notice that

$$
\int_{x_{i}}^{x_{i+1}}\left(x-x_{i}\right) \phi(x) d x=\Delta x \psi\left(x_{i+1}\right)-\int_{x_{i}}^{x_{i+1}} \psi(x) d x,
$$

where $\psi$ is an antiderivative of $\phi$. Thus we get

$$
A=\sum_{i} \frac{1}{\Delta t}\left[\frac{U_{i+1}^{n+1}-U_{i}^{n+1}-U_{i+1}^{n}+U_{i}^{n}}{\Delta x}\right] \Delta x \Delta \psi_{i+1 / 2},
$$

with $\Delta \psi_{i+1 / 2}:=\psi\left(x_{i+1}\right)-\frac{1}{\Delta x} \int_{x_{i}}^{x_{i+1}} \psi(x) d x$. Moreover, by doing translations of indices we get

$$
A=\sum_{i} \frac{1}{\Delta t}\left[U_{i}^{n+1}-U_{i}^{n}\right]\left[\Delta \psi_{i-1 / 2}-\Delta \psi_{i+1 / 2}\right] .
$$

Next, using that $U_{i}^{n}$ is bounded we get from (1.20) that

$$
\left|U_{i}^{n+1}-U_{i}^{n}\right| \leq 2 \frac{\Delta t}{\Delta x}\left(\left\|F^{+}(U)\right\|_{\infty}+\left\|F^{-}(U)\right\|_{\infty}+\left\|g h^{2}\right\|_{\infty}\right) .
$$

Moreover we notice that

$$
\left|\Delta \psi_{i-1 / 2}-\Delta \psi_{i+1 / 2}\right| \leq \Delta x^{2} \operatorname{Lip}(\phi),
$$

which enables us to get

$$
|A| \leq 2 C \Delta x \operatorname{Lip}(\phi) \sum_{i} \mathbf{1}_{\operatorname{dist}\left(x_{i}, \operatorname{supp} \phi\right) \leq \Delta x} \leq C^{\prime},
$$


with $C^{\prime}>0$ a constant depending on $\phi$. Next, from (A.107), we use (1.20) and get

$$
B=-\sum_{i} \frac{1}{\Delta x}\left[F_{i+1 / 2-}-F_{i-1 / 2+}\right] \Delta x \phi_{i+1 / 2},
$$

with $\phi_{i+1 / 2}:=\frac{1}{\Delta x} \int_{x_{i}}^{x_{i+1}} \phi(x) d x$. Using (1.21)-(1.23) we get

$$
\begin{aligned}
B & =-\sum_{i}\left[\mathcal{F}_{i+1 / 2}-\mathcal{F}_{i-1 / 2}\right] \phi_{i+1 / 2}-\sum_{i}\left[-S_{i+1 / 2-}-S_{i-1 / 2+}\right] \phi_{i+1 / 2} \\
& =-\sum_{i} \mathcal{F}_{i+1 / 2}\left[\phi_{i+1 / 2}-\phi_{i+3 / 2}\right]+\sum_{i}\left[S_{i+1 / 2-}+S_{i-1 / 2+}\right] \phi_{i+1 / 2} .
\end{aligned}
$$

We have

$$
\left|\phi_{i+1 / 2}-\phi_{i+3 / 2}\right| \leq \Delta x \operatorname{Lip}(\phi)
$$

and

$$
\left|S_{i+1 / 2-}+S_{i-1 / 2+}\right| \leq v_{m}^{2}\left(\left|z_{i+1}-z_{i}\right|+\left|z_{i}-z_{i-1}\right|\right) .
$$

We thus conclude that $B$ is also bounded since $\partial_{x} z_{\Delta}$ is bounded in $L_{l o c}^{1}$.

Lemma A.9. With the assumptions of Theorem 1.1, let $U_{\Delta}=\left(h_{\Delta}, h_{\Delta} u_{\Delta}\right)$ be the approximate solution (1.33), and $\widetilde{S}_{\Delta}$ be the approximate source defined by (3.6). We assume that there exists $U$ such that $U_{\Delta}$ tends to $U$ a.e. as $\Delta x, \Delta t \rightarrow 0$. Then

$\forall \phi(t, x) \in \mathcal{D}\left(\mathbb{R}^{2}\right), \quad \iint \widetilde{S}_{\Delta}(t, x) \phi(t, x) d t d x \underset{\Delta x, \Delta t \rightarrow 0}{\longrightarrow} \iint S(t, x) \phi(t, x) d t d x$,

with $S(t, x)=\left(\begin{array}{c}0 \\ -g h \partial_{x} z\end{array}\right)$.

Proof. Let $\phi(t, x) \in \mathcal{D}\left(\mathbb{R}^{2}\right)$. We compute the integral

$$
\iint \widetilde{S}_{\Delta}(t, x) \phi(t, x) d t d x=\sum_{n=0}^{\infty} \sum_{i=-\infty}^{\infty} \Delta t\left(S_{i+1 / 2-}+S_{i-1 / 2+}\right) \phi_{i}^{n},
$$

with $\phi_{i}^{n}=\frac{1}{\Delta t} \frac{1}{\Delta x} \int_{t_{n}}^{t_{n+1}} \int_{x_{i-1 / 2}}^{x_{i+1 / 2}} \phi(t, x) d t d x$. Then we perform a translation of the index $i$ and get

$$
\begin{aligned}
& \sum_{n} \sum_{i} \Delta t\left(S_{i+1 / 2-}+S_{i-1 / 2+}\right) \phi_{i}^{n} \\
= & \sum_{n} \Delta t \sum_{i} S_{i+1 / 2-} \phi_{i}^{n}+\sum_{n} \Delta t \sum_{i} S_{i+1 / 2+} \phi_{i+1}^{n} .
\end{aligned}
$$


Then we notice that $\left|\phi_{i+1}^{n}-\phi_{i}^{n}\right| \leq \operatorname{Lip}(\phi) \Delta x$ and we obtain that

$$
\iint \widetilde{S}_{\Delta} \phi d t d x-\sum_{n} \sum_{i} \Delta t\left(S_{i+1 / 2-}+S_{i+1 / 2+}\right) \phi_{i}^{n} \rightarrow 0 .
$$

Next, for $\Delta x,\left|z_{i+1}-z_{i}\right|$ small enough, we have on the one hand using (1.43)

$$
\begin{aligned}
S_{i+1 / 2-}^{h u} & =g \frac{h_{i+1 / 2-}^{2}}{2}-g \frac{h_{i}^{2}}{2} \\
& =g \frac{\left(h_{i}+z_{i}-z_{i+1 / 2}\right)^{2}}{2}-g \frac{h_{i}^{2}}{2} \\
& =g\left(z_{i}-z_{i+1 / 2}\right)\left(h_{i}+\frac{z_{i}-z_{i+1 / 2}}{2}\right) .
\end{aligned}
$$

On the other hand, we have similarly

$$
S_{i+1 / 2+}^{h u}=g \frac{h_{i+1}^{2}}{2}-g \frac{h_{i+1 / 2+}^{2}}{2}=-g\left(z_{i+1}-z_{i+1 / 2}\right)\left(h_{i+1}+\frac{z_{i+1}-z_{i+1 / 2}}{2}\right) .
$$

Moreover noticing that $h_{i+1}=h_{i}+\left(h_{i+1}-h_{i}\right)$, with (A.120), (A.121) we get

$$
\begin{aligned}
& \sum_{n} \sum_{i} \Delta t\left(S_{i+1 / 2-}^{h u}+S_{i+1 / 2+}^{h u}\right) \phi_{i}^{n} \\
& =-\sum_{n} \sum_{i} \Delta t g\left(z_{i+1}-z_{i}\right) h_{i}^{n} \phi_{i}^{n}+\sum_{n} \sum_{i} \Delta t \Delta x R_{i}^{n} \phi_{i}^{n},
\end{aligned}
$$

with

$\Delta x R_{i}^{n}=-g\left(z_{i+1}-z_{i+1 / 2}\right)\left(h_{i+1}^{n}-h_{i}^{n}\right)+g \frac{\left(z_{i}-z_{i+1 / 2}\right)^{2}}{2}-g \frac{\left(z_{i+1}-z_{i+1 / 2}\right)^{2}}{2}$.

The last term in the RHS of (A.122) tends to 0 because of (1.36), the Cauchy Schwarz inequality and the bound (2.3). The first term in the RHS of (A.122) converges to the source term since

$$
\begin{aligned}
& -\sum_{n} \sum_{i} \Delta \operatorname{tg}\left(z_{i+1}-z_{i}\right) h_{i}^{n} \phi_{i}^{n} \\
& \simeq \iint-g \frac{\mathrm{d} z_{\Delta}(x)}{\mathrm{d} x} h_{\Delta}(t, x) \phi(t, x) d t d x \rightarrow \iint-g \frac{\mathrm{d} z(x)}{\mathrm{d} x} h(t, x) \phi(t, x) d t d x .
\end{aligned}
$$

The convergence holds because we supposed $h_{\Delta} \rightarrow h$ a.e., and $\frac{\mathrm{d} z_{\Delta}}{\mathrm{d} x} \rightarrow \frac{\mathrm{d} z}{\mathrm{~d} x}$ in $L_{l o c}^{1}$. This concludes the proof of the lemma. 


\section{References}

[1] D. Amadori, L. Gosse, Transient L1 error estimates for well-balanced schemes on non-resonant scalar balance laws, J. Diff. Eq. 255 (2013), 469-502.

[2] D. Amadori, L. Gosse, Stringent error estimates for one-dimensional, space-dependent $2 \times 2$ relaxation systems, Ann. IHP (C) Nonlinear Analysis 33 (2016), 621-654.

[3] N. Andrianov, Performance of numerical methods on the non-unique solution to the Riemann problem for the shallow water equations, Intern. J. Num. Meth. Fluids 47 (2005), 825-831.

[4] E. Audusse, F. Bouchut, M.-O. Bristeau, R. Klein, B. Perthame, A fast and stable well-balanced scheme with hydrostatic reconstruction for shallow water flows, SIAM J. Sci. Comput. 25 (2004), 2050-2065.

[5] E. Audusse, F. Bouchut, M.-O. Bristeau, J. Sainte-Marie, Kinetic entropy inequality and hydrostatic reconstruction scheme for the SaintVenant system, Math. Comput. 85 (2016), 2815-2837.

[6] E. Audusse, M.-O. Bristeau, A well-balanced positivity preserving second-order scheme for Shallow Water flows on unstructured meshes, J. Comput. Phys. 206 (2005), 311-333.

[7] E. Audusse, M.-O. Bristeau, B. Perthame, J. Sainte-Marie, A multilayer Saint-Venant system with mass exchanges for Shallow Water flows. Derivation and numerical validation, ESAIM: M2AN 45 (2011), 169-200.

[8] F. Berthelin, Convergence of flux vector splitting schemes with single entropy inequality for hyperbolic systems of conservation laws, Numer. Math. 99 (2005), 585-604.

[9] F. Berthelin, F. Bouchut, Kinetic invariant domains and relaxation limit from a BGK model to isentropic gas dynamics, Asymptotic Analysis 31 (2002), 153-176.

[10] F. Berthelin, F. Bouchut, Relaxation to isentropic gas dynamics for a BGK system with single kinetic entropy, Meth. Appl. Anal. 9 (2002), 313-327.

[11] C. Berthon, C. Chalons, A fully well-balanced, positive and entropysatisfying Godunov-type method for the shallow-water equations, Math. Comput. 85 (2016), 1281-1307. 
[12] C. Berthon, F. Marche, A positive preserving high order VFRoe scheme for shallow water equations: a class of relaxation schemes, SIAM J. Scientific Comput. 30 (2008), 2587-2612.

[13] F. Bouchut, Entropy satisfying flux vector splittings and kinetic BGK models, Numer. Math. 94 (2003), 623-672.

[14] F. Bouchut, H. Frid, Finite difference schemes with cross derivatives correctors for multidimensional parabolic systems, J. Hyp. Diff. Eq. 3 (2006), 27-52.

[15] F. Bouchut, X. Lhébrard, A multi well-balanced scheme for the shallow water MHD system with topography, Numer. Math. 136 (2017), 875-905.

[16] F. Bouchut, T. Morales De Luna, A subsonic-well-balanced reconstruction scheme for shallow water flows, Siam J. Numer. Anal. 48 (2010),1733-1758.

[17] F. Bouchut, V. Zeitlin, A robust well-balanced scheme for multi-layer shallow water equations, Discrete and Continuous Dynamical Systems B, 13 (2010), 739-758.

[18] M.J. Castro, A. Pardo, C. Parés, Well-balanced numerical schemes based on a generalized hydrostatic reconstruction technique, Math. Models Methods Appl. Sci. 17 (2007), 2065-2113.

[19] F. Coquel, K. Saleh, N. Seguin, A robust and entropy-satisfying numerical scheme for fluid flows in discontinuous nozzles, Math. Models Meth. Appl. Sci. 24 (2014), 2043-2083.

[20] V. Desveaux, M. Zenk, C. Berthon, C. Klingenberg, Well-balanced schemes to capture non-explicit steady states: Ripa model, Math. Comp. 85 (2016), 1571-1602.

[21] R. J. DiPerna, Convergence of approximate solutions to conservation laws, Archive Rational Mech. Anal. 82 (1983), 27-70.

[22] L. Gosse, A.E. Tzavaras, Convergence of relaxation schemes to the equations of elastodynamics, Math. Comp. 70 (2001), 555-577.

[23] P.-L. Lions, B. Perthame, P. Souganidis, Existence and stability of entropy solutions for the hyperbolic systems of isentropic gas dynamics in Eulerian and Lagrangian coordinates, Comm. Pure Applied Math. 49 (1996), 599-638. 
[24] C. Parés, Numerical methods for nonconservative hyperbolic systems: a theoretical framework, SIAM J. Numer. Anal. 44 (2006), 300-321.

[25] B. Perthame, C. Simeoni, A kinetic scheme for the Saint Venant system with a source term, Calcolo 38 (2001), 201-231.

[26] B. Perthame, C. Simeoni, Convergence of the upwind interface source method for hyperbolic conservation laws, In Hyperbolic problems: theory, numerics, applications, 61-78, Springer, 2003.

[27] L. Tartar, Compensated compactness and applications to partial differential equations, In Nonlinear analysis and mechanics, Heriot-Watt symposium, vol. 4, 136-211, Pitman, 1979.

[28] A. Vasseur, Well-posedness of scalar conservation laws with singular sources, Meth. Appl. Anal. 9 (2002), 291-312.

[29] G. Warnecke N. Andrianov, On the solution to the riemann problem for the compressible duct flow, SIAM J. Appl. Math. 64 (2004), 878-901. 